\title{
Early Detection Methods for Silicosis in Australia and Internationally: A Review of the Literature
}

\author{
Emma K. Austin ${ }^{1, *}$, Carole James ${ }^{1,2}$ and John Tessier ${ }^{2}$ \\ 1 Centre for Resources Health and Safety, College of Health, Medicine and Wellbeing, University of Newcastle, \\ Callaghan, NSW 2308, Australia; Carole.James@newcastle.edu.au \\ 2 School of Health Sciences, College of Health, Medicine and Wellbeing, University of Newcastle, \\ Callaghan, NSW 2308, Australia; John.Tessier@newcastle.edu.au \\ * Correspondence: Emma.Austin@newcastle.edu.au
}

Citation: Austin, E.K.; James, C.;

Tessier, J. Early Detection Methods for Silicosis in Australia and Internationally: A Review of the Literature. Int. J. Environ. Res. Public Health 2021, 18, 8123. https:// doi.org/10.3390/ijerph18158123

Academic Editor: Ivo Iavicoli

Received: 25 May 2021

Accepted: 7 July 2021

Published: 31 July 2021

Publisher's Note: MDPI stays neutral with regard to jurisdictional claims in published maps and institutional affiliations.

Copyright: (c) 2021 by the authors. Licensee MDPI, Basel, Switzerland. This article is an open access article distributed under the terms and conditions of the Creative Commons Attribution (CC BY) license (https:// creativecommons.org/licenses/by/ $4.0 /)$.

\begin{abstract}
Pneumoconiosis, or occupational lung disease, is one of the world's most prevalent workrelated diseases. Silicosis, a type of pneumoconiosis, is caused by inhaling respirable crystalline silica (RCS) dust. Although silicosis can be fatal, it is completely preventable. Hundreds of thousands of workers globally are at risk of being exposed to RCS at the workplace from various activities in many industries. Currently, in Australia and internationally, there are a range of methods used for the respiratory surveillance of workers exposed to RCS. These methods include health and exposure questionnaires, spirometry, chest X-rays, and HRCT. However, these methods predominantly do not detect the disease until it has significantly progressed. For this reason, there is a growing body of research investigating early detection methods for silicosis, particularly biomarkers. This literature review summarises the research to date on early detection methods for silicosis and makes recommendations for future work in this area. Findings from this review conclude that there is a critical need for an early detection method for silicosis, however, further laboratory- and field-based research is required.
\end{abstract}

Keywords: silicosis; pneumoconiosis; early detection; respiratory surveillance

\section{Introduction}

Silicosis is an incurable, sometimes fatal, but completely preventable lung disease caused by exposure to respirable crystalline silica (RCS). Worldwide, thousands of workers in a range of industries are at risk of developing silicosis. Early detection of silicosis is vital to identify the disease at a pre-clinical stage to allow interventions that improve outcomes for workers, while investigating inadequacies in workplace control practices. Currently, international respiratory surveillance includes health and exposure questionnaires, spirometry, X-rays, and high-resolution computed tomography (HRCT). However, some of these techniques are unable to detect silicosis at an early stage. This review investigates current respiratory surveillance for silicosis and explores potential opportunities for alternative early detection methods, particularly biomarkers and exhaled breath condensate (EBC).

Pneumoconioses are a group of non-malignant parenchymal (interstitial) lung diseases caused by inhaling dust particles [1,2]. Worldwide, one of the most common work-related injuries is pneumoconiosis, specifically caused by exposure to RCS [3]. Indeed, in China, pneumoconiosis is the most prevalent occupational disease [4]. Recent years have seen a resurgence of certain types of pneumoconiosis, particularly in the United States [5] and Australia [6].

The three primary types of pneumoconiosis are asbestosis, coal workers' pneumoconiosis (CWP), and silicosis [6]. Silicosis is a fibrotic lung disease caused by inhaling RCS [7]. For both developed and developing countries, silicosis is a major cause of mortality and morbidity [8]. Silicosis is highly prevalent in low- and middle-income countries, although the true extent is likely underreported due to poor respiratory surveillance [7]. 
Australia has recently experienced a surge in silicosis cases as a result of growth in the manufactured stone industry. In 2019, there were an estimated 350 cases of silicosis in Australia, with 100 cases identified between September and December [9]. Given that over 500,000 Australians are exposed to RCS in the workplace annually [10], silicosis has the potential for huge socioeconomic impact. In addition to silicosis, in 2015, the Queensland government received its first report of CWP in over 30 years [6], prompting the Queensland Government Department of Natural Resources, Mines and Energy (DNRME) to conduct a review of the health assessment performed under the Queensland Coal Mine Workers' Health Scheme [11].

Industries where occupational exposure to RCS is prominent include manufactured stone, stone masons, coal mining, denim blasting, dental technicians, and other various trades [12]. Table 1 demonstrates the broad range of workplaces where RCS exposure can occur. In addition, exposure is also possible via background environmental conditions [13] and volcanic eruptions [14].

Table 1. Common operations or tasks that involve exposure to free crystalline silica $[7,15,16]$.

\begin{tabular}{|c|c|}
\hline Activity & Industries or Occupational Activities \\
\hline Drilling & Construction, quarrying and related milling, mining and related milling, tunneling \\
\hline Breaking and crushing & Construction, quarrying and related milling, mining and related milling, tunneling \\
\hline Cutting & $\begin{array}{l}\text { Arts, crafts, and sculpture, jewelry, construction, quarrying and related milling, } \\
\text { grindstone production }\end{array}$ \\
\hline Abrasive blasting and sand blasting & $\begin{array}{l}\text { Boiler scaling, production of dental material, metal products, automobile repair (removal of } \\
\text { paint and rust), arts, crafts, and sculpture, shipbuilding and repair, foundries, construction, } \\
\text { quarrying and related milling, production of denim jeans, tombstone production }\end{array}$ \\
\hline Grinding & Arts, crafts, and sculpture, jewelry, construction, quarrying and related milling \\
\hline Sanding & Automobile repair (removal of paint and rust), construction \\
\hline Excavation and digging & $\begin{array}{l}\text { Agriculture, construction, quarrying and related milling, mining and related } \\
\text { milling, tunneling }\end{array}$ \\
\hline Hammering & Boiler scaling, construction \\
\hline Casting and moulding & Jewelry, foundries, ceramics \\
\hline $\begin{array}{l}\text { Furnace installation and repair } \\
\text { (refractory materials) }\end{array}$ & Iron and steel mills, foundries, glass \\
\hline
\end{tabular}

Cleaning (dry sweeping and brushing, pressurised air blowing)

Construction, arts, crafts, and sculpture, jewelry

Polishing and buffing

Production of dental material, arts, crafts, and sculpture, jewelry

Worldwide, silica (silicon dioxide) is a naturally occurring and abundant mineral, forming the major element of most rocks and soils $[7,17,18]$. There are non-crystalline and crystalline forms of silicon dioxide, with only crystalline forms causing pneumoconiosis. Silica dust is generated in the workplace in a range of industries. Mechanical processes in the workplace, such as sawing, crushing, drilling, polishing, cutting, or grinding of natural stone or manufactured products, produce the harmful dust. Respirable particles are dust particles that are so small they are not visible [17]. In addition to silicosis, RCS is associated with a number of diseases, including lung cancer, chronic obstructive pulmonary disease (COPD), tuberculosis, scleroderma, rheumatoid arthritis, autoimmune diseases (AIDs), and chronic kidney disease $[19,20]$. Some patients with silicosis are susceptible to also developing tuberculosis (silicotuberculosis) [21].

The symptoms of silicosis differ according to the stage and severity of the disease. Simple silicosis may be asymptomatic and incidentally diagnosed during routine respiratory surveillance. The most commonly recognised form of the disease is chronic silicosis, which usually develops after exposure to low concentrations of silica dust for 10 or more years [8]. 
Symptoms of chronic silicosis may include cough and shortness of breath. Accelerated silicosis shares some clinical features with chronic silicosis, although it often progresses more rapidly and develops 5-10 years after initial exposure [15]. Acute silicosis is rare and develops after exposure to high concentrations of silica dust for a period of a few weeks to five years [7]. Symptoms of acute silicosis include dyspnoea, dry cough, fever, fatigue, and weight loss, with respiratory failure and death often occurring within a few months [7]. For all three forms of silicosis, the rate of development is dependent on the surface characteristics of the RCS particles and the intensity and duration of exposure [22].

Respiratory surveillance (also referred to as occupational lung disease screening) in Australia and overseas has common elements, although differences do occur due to available technology and the cost of surveillance. Exposure history and respiratory symptom questionnaires constitute the first step in respiratory health surveillance. Spirometry is a second commonly used method. Together with questionnaires and spirometry, medical imaging — commonly radiography (X-ray) —is used for the diagnosis, surveillance, and screening of occupational lung disease. In addition, various HRCT protocols are a final step in the surveillance process.

Pneumoconiosis appears as small dot-like opacities on chest X-rays and HRCT, with the shape, size, and quantity of these opacities graded to represent the severity of the disease [6]. For X-rays, this grading is typically conducted using the International Labour Office (ILO) Classification System [23] and, depending on the organisational/regulatory requirements, may be interpreted by one or multiple qualified B readers [15].

Specifically, the US follows these standard methods of respiratory surveillance, and there appears to have been little change in this for some time, although some new monitoring techniques continue to be developed [24]. NIOSH [24] outline occupational respiratory surveillance in the US to include questionnaires, radiography, spirometry, and biomarkers. Surveillance has two phases: (i) an initial medical examination that includes history, physical examination, respiratory and cardiovascular examinations, chest $\mathrm{X}$-ray, and pulmonary function testing (FVC and FEV (1 s)); and (ii) periodic medical examinations on an annual basis [24]. These methods, together with sputum cytology and tuberculin skin tests, are identified as the specific medical tests and examinations for the Occupational Safety and Health Administration (OSHA) regulated substances [25]. As identified by this review, NIOSH also recognises that an optimal method for the early detection of pneumoconiosis is yet to be developed.

Following the unexpected reporting in 2015 of the first case of CWP in over 30 years in Australia, there has been a renewal of the Coal Mine Workers' Health Scheme [6]. Subsequently, the highest number of cases of CMDLD ever diagnosed in Queensland, Australia has been recorded [6]. This resurgence highlights the need for regular respiratory surveillance with a high level of sensitivity.

Most silicosis cases are not diagnosed at an early stage, as the initial phase of the disease is typically asymptomatic [21] and is often undetectable with spirometry and X-ray. Specifically, silicosis can present a diagnostic challenge due to its radiological resemblance and clinical overlap with sarcoidosis, pulmonary tuberculosis, and neoplastic lesions [26]. In addition, barriers to early diagnosis include a lack of a suitable biomarker, poor health-seeking behavior, insufficient occupational healthcare services at workplaces, particularly in developing countries, and unorganised sectors [21]. Impediments to early diagnosis also include a lack of education and understanding of the level of risk associated with RCS and the limitations of the current methods of initial medical screening (i.e., spirometry and X-ray). In Australia specifically, cultural barriers of a predominantly young, migrant workforce, the growth of the manufactured stone industry, and a historical lack of regulation are suggested to have contributed to a resurgence of silicosis in some populations. Surveillance is needed long-term, even after retirement and cessation of exposure, due to the long latency of silicosis.

Compounding the issue of exposure to RCS is that X-ray and HRCT present the concern of giving a regular radiation dose to workers. For example, in NSW, workers need 
to be scanned every year for their whole career. Regulators have a duty of care not to expose workers to an ongoing, annual dose of radiation, although it may be argued that the level of radiation is incidental, and must be weighed against the opportunity for a more sensitive test that reliably detects disease [19].

Despite efforts to establish and maintain best practices, respiratory surveillance continues to be a disparate process [27]. Standardisation of the process is required in order to protect workers exposed to RCS.

Despite the acknowledgment that improved detection methods other than spirometry, $\mathrm{X}$-ray, and HRCT are needed, knowledge gaps remain around alternatives. Although research has been conducted into EBC and biomarkers as methods for detecting silicosis, these techniques have not been validated, and remain at an investigative stage. In addition, there is inconclusive evidence as to which biomarker(s) most effectively capture silicosis.

The literature review was directed by the following research questions:

- What methods are currently used in respiratory surveillance for occupational lung disease? Have they been validated?

- What alternative methods exist or are under investigation, and what evidence is there for the effectiveness of these methods?

- Is there evidence to support conducting a prospective cohort study to test the validity of alternative methods of early detection of silicosis?

The overarching objective of this review is to inform changes to respiratory surveillance with the global goal to reduce the prevalence of silicosis and improve the prognosis of workers who develop silicosis. Although the search did return a large number of studies that investigated treatments, including murine experiments and investigations of DNA, treatments for silicosis are not a focus of this review.

\section{Materials and Methods}

The review involved three separate search strategies: a scoping literature review of peer-reviewed articles, a search of the grey literature, and a search of websites and online material. In addition, the research team consulted with leading academics and regulatory professionals in Australia and overseas to gain insights into the current prevalence of silicosis and screening methods.

\subsection{Scoping Review}

The scoping review was conducted according to the PRISMA-ScR framework. PRISMA$\mathrm{ScR}$ is a systematic approach to assist with mapping evidence on a topic and identifying the main concepts, theories, and knowledge gaps relevant to that topic [28,29].

An initial browsing search of the online database MEDLINE was completed to familiarise the researchers with the key search terms. The scoping review search was conducted in the online library databases Scopus, Embase, and CINAHL. Using the keyword search function, search terms were: "silicosis" or "pneumoconiosis" or "black lung" or "respiratory fibrosis" or "dust disease", together with "monitor" or "early detect" or "mass screening" or "screen" (truncation and proximity searching were applied to some terms). The search was limited to articles written in English and published since 2010. Articles were catalogued and screened using the referencing software Endnote and the web-based software platform Covidence, which streamlines the production of reviews. The reference lists of included articles were also searched. The search was conducted in March 2020.

\subsection{Grey Literature}

When searching grey literature, it was necessary to keep the search terms more broad than when searching peer-reviewed journal databases. Google Scholar was searched with the terms "silicosis" and "early detection" and "screening". The records were presented according to relevancy; the first 200 records were screened, and those that were appropriate were included in the review [30]. The Mednar database was searched with the search term "silicosis". The first 200 records were screened, and relevant documents were included in 
the review. Mednar conducts a comprehensive search across medical societies, the National Institute of Health resources, US government websites, and patents. The OpenTrials database was searched to find clinical trials specifically relating to silicosis and early detection methods.

\subsection{Websites, Industry, Government, and Regulators}

When searching websites, it is optimal to maintain generic and overarching search terms, as the search relies on the website's own search engine. Websites searched included regulatory bodies, industry organisations, and government websites in Australia and internationally.

\section{Results}

The findings from the three search methods are synthesised below. The database search for the review returned 1751 articles. After 46 duplicates were removed, the titles and abstracts of 1705 articles were screened for relevance, of which 122 progressed to full-text screening. This final screening process determined that 52 articles were eligible for the final scoping review. Figure 1 shows the screening process for the scoping review. The final 52 articles included in the scoping review are summarised in Table 2.

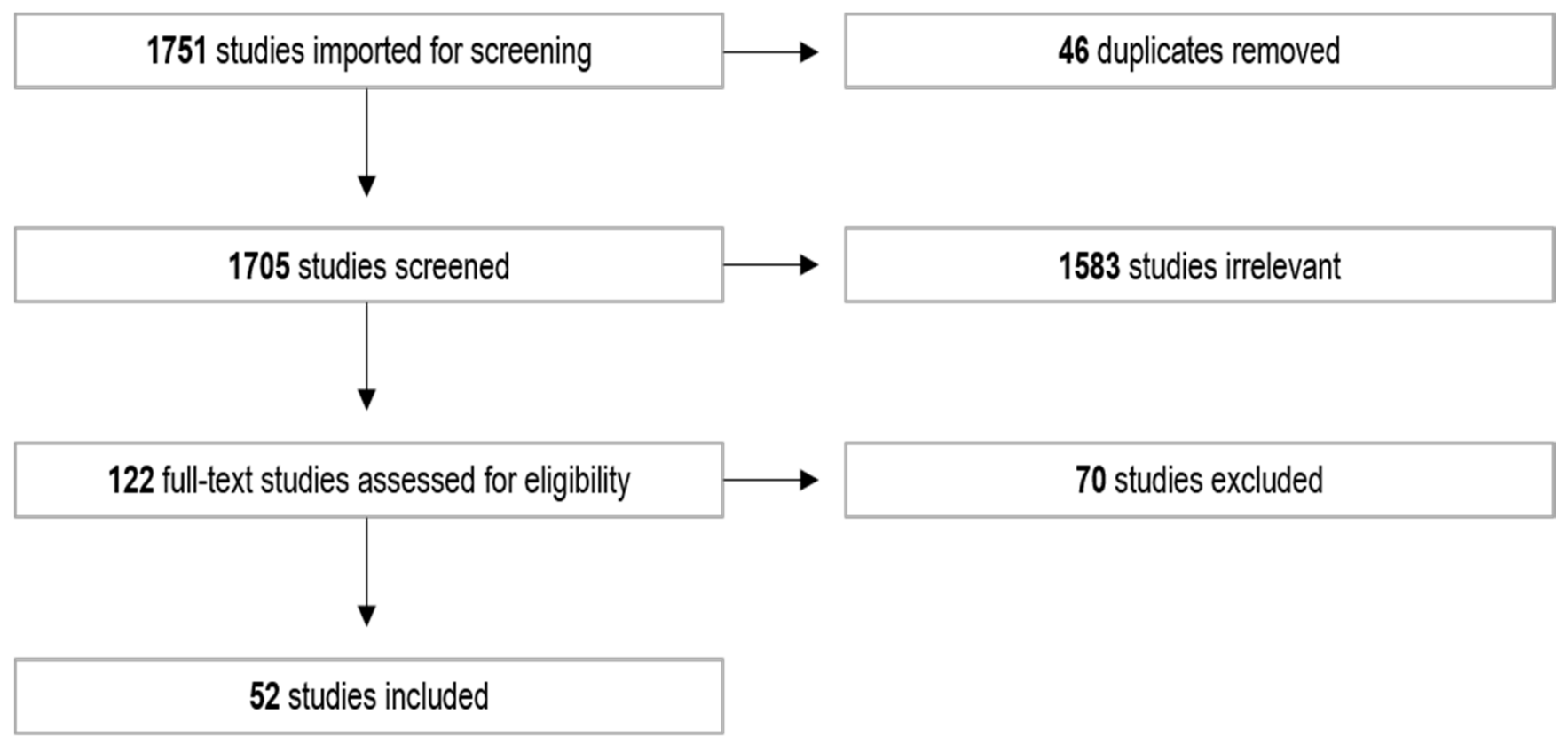

Figure 1. PRISMA flow chart showing the screening process for the scoping review. 
Table 2. Extraction table of the 52 articles included in the scoping review.

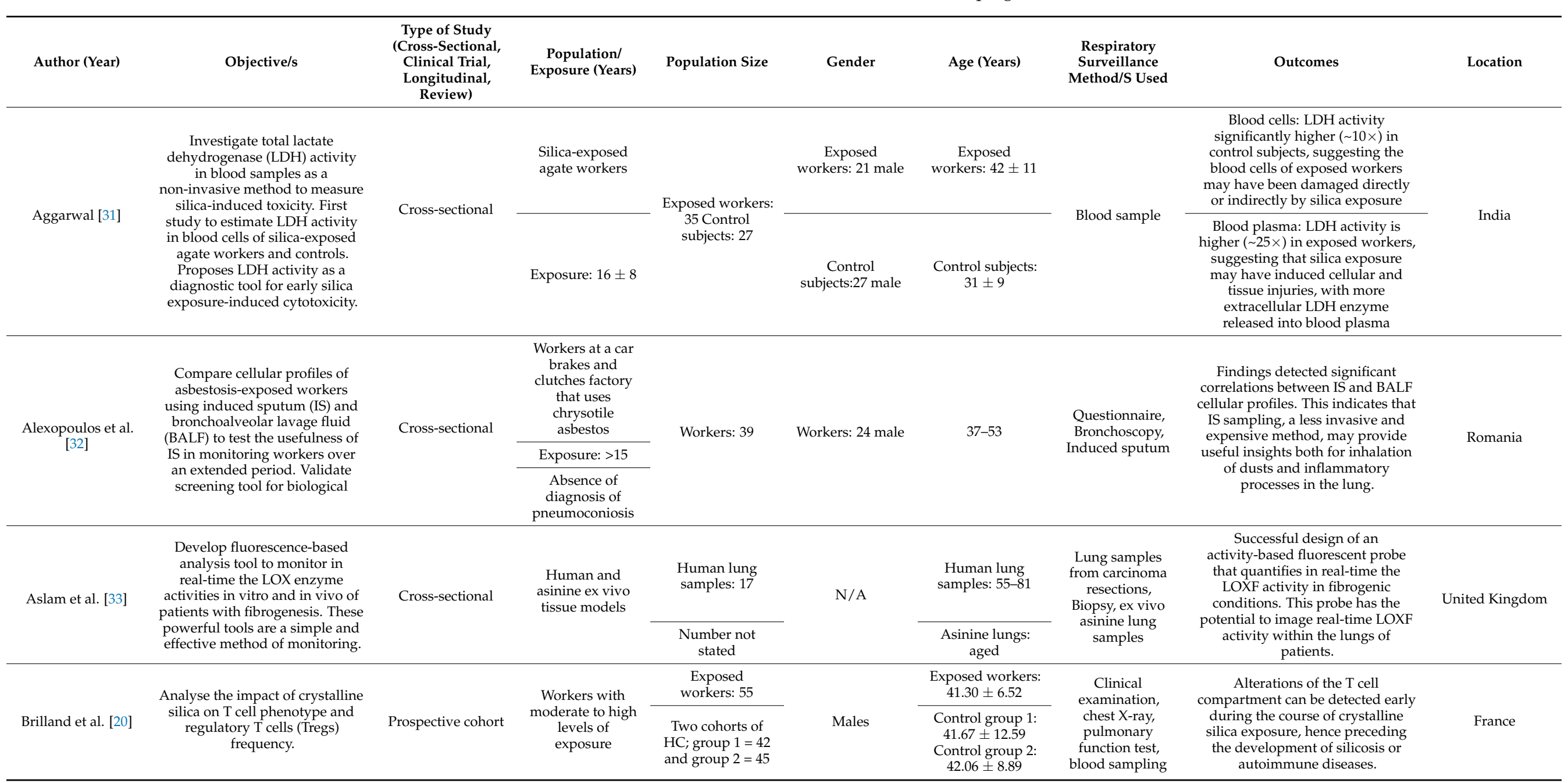


Table 2. Cont.

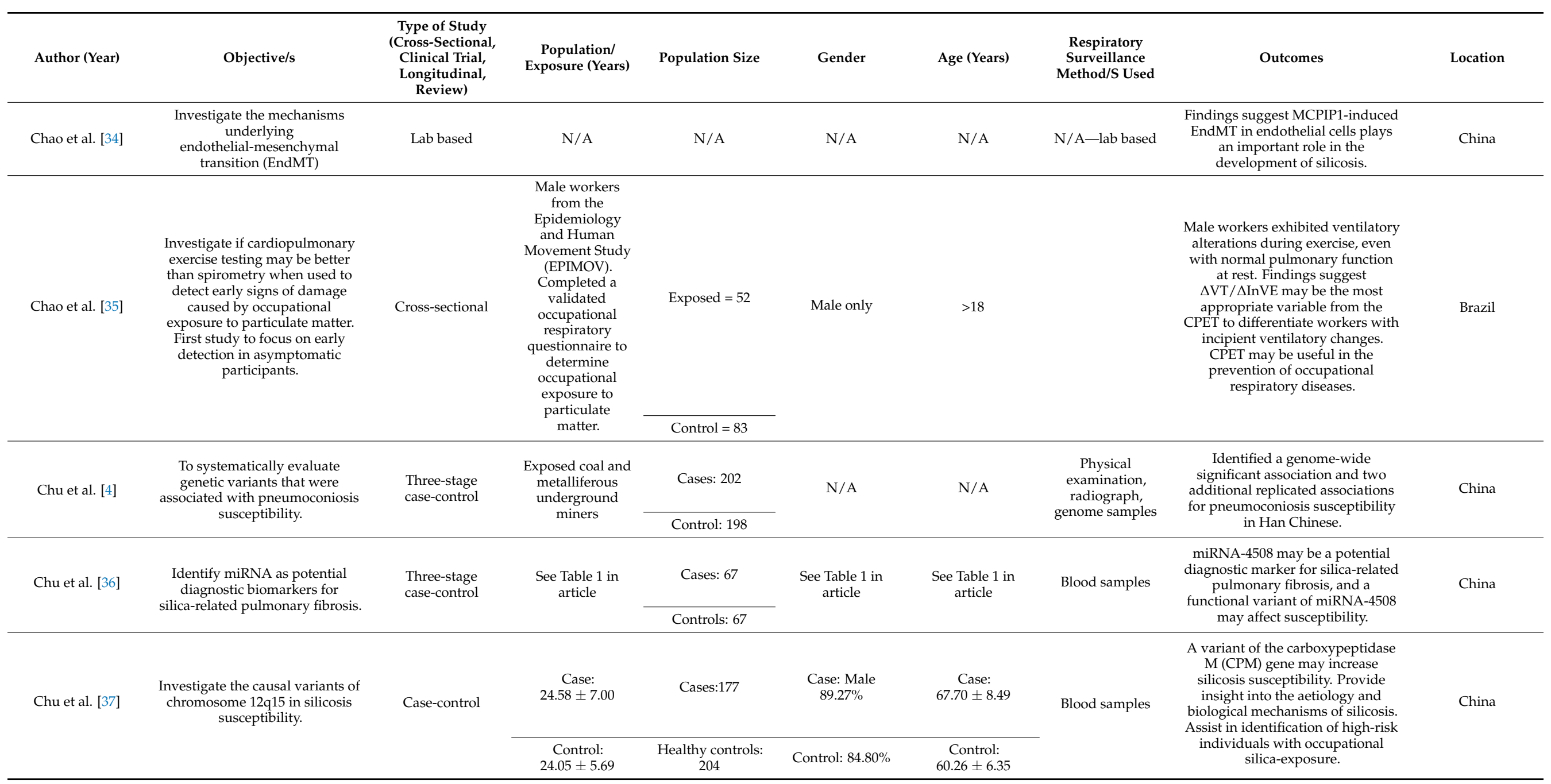


Table 2. Cont

\begin{tabular}{|c|c|c|c|c|c|c|c|c|c|}
\hline Author (Year) & Objective/s & $\begin{array}{c}\text { Type of Study } \\
\text { (Cross-Sectional, } \\
\text { Clinical Trial, } \\
\text { Longitudinal, } \\
\text { Review) }\end{array}$ & $\begin{array}{c}\text { Population/ } \\
\text { Exposure (Years) }\end{array}$ & Population Size & Gender & Age (Years) & $\begin{array}{l}\text { Respiratory } \\
\text { Surveillance } \\
\text { Method/S Used }\end{array}$ & Outcomes & Location \\
\hline $\begin{array}{c}\text { Codorean et al. } \\
\text { [38] }\end{array}$ & $\begin{array}{l}\text { Perform exploratory study on } \\
\text { peripheral whole-blood to } \\
\text { analyse early effects of exposure } \\
\text { in coal fired power plants. }\end{array}$ & Cross-sectional & $\begin{array}{l}\text { Three groups: } 10 \\
\text { years; } 20 \text { years; } \\
\text { control }\end{array}$ & N/A & $\mathrm{N} / \mathrm{A}$ & N/A & Blood samples & $\begin{array}{l}\text { This method is non-invasive and } \\
\text { rapid and could be a useful tool } \\
\text { in identifying early hazard before } \\
\text { it is diagnosed clinically. }\end{array}$ & Romania \\
\hline Corradi et al. [39] & $\begin{array}{l}\text { Review EBC studies that } \\
\text { investigate exposure and effect } \\
\text { biomarkers in lung disease, } \\
\text { particularly toxic metals }\end{array}$ & Review & N/A & N/A & N/A & N/A & $\begin{array}{c}\text { Exhaled breath } \\
\text { condensate (EBC) }\end{array}$ & $\begin{array}{l}\text { Exhaled breath biomarkers have } \\
\text { been shown to be capable of } \\
\text { detecting and monitoring } \\
\text { diseases of the respiratory } \\
\text { system. }\end{array}$ & N/A \\
\hline $\begin{array}{c}\text { Cox and Lynch } \\
\text { [40] }\end{array}$ & $\begin{array}{c}\text { Provide review of recent } \\
\text { developments in medical } \\
\text { imaging of environmental lunch } \\
\text { disease. }\end{array}$ & Review & N/A & N/A & $\mathrm{N} / \mathrm{A}$ & N/A & Medical imaging & $\begin{array}{l}\text { Medical imaging is useful in the } \\
\text { diagnosis, epidemiological study } \\
\text { and management of occupational } \\
\text { lung disease. Studies that } \\
\text { compare HRCT with film-screen } \\
\text { radiography found CT was more } \\
\text { sensitive. }\end{array}$ & $\mathrm{N} / \mathrm{A}$ \\
\hline Dinescu et al. [41] & $\begin{array}{l}\text { Identify correlations between } \\
\text { electrocardiographic and } \\
\text { echocardiographic changes in } \\
\text { patients with silicosis prior to } \\
\text { chronic pulmonary heart disease } \\
\text { occurring. }\end{array}$ & $\begin{array}{l}\text { Prospective, } \\
\text { descriptive, } \\
\text { analytical }\end{array}$ & N/A & $\begin{array}{l}\text { Cases: } 67 \\
\text { Control: } 25\end{array}$ & N/A & $\begin{array}{l}\text { Cases: } 477-8 \\
\text { years }\end{array}$ & $\begin{array}{l}\text { Electrocardiograph, } \\
\text { echocardiograph }\end{array}$ & $\begin{array}{l}\text { Values of the right heart } \\
\text { echocardiographic parameters at } \\
\text { the upper limit of normality are } \\
\text { early markers for cardiovascular } \\
\text { damage in patients with silicosis. }\end{array}$ & Romania \\
\hline Doganay et al. [3] & $\begin{array}{l}\text { Assess MDCT findings of } \\
\text { silicosis in denim sandblasters } \\
\text { and define the role of MDCT in } \\
\text { the early detection of silicosis. }\end{array}$ & Cross-sectional & $\begin{array}{c}\begin{array}{c}\text { Denim } \\
\text { sandblasters }\end{array} \\
1.0-5.2 \text { years } \\
3.7 \pm 1.4 \\
\end{array}$ & $\begin{array}{l}12 \text { male patients } \\
\text { admitted to a } \\
\text { pulmonary } \\
\text { outpatient clinic } \\
\text { between } \\
\text { April-December } \\
\text { 2009. } \\
\end{array}$ & Male only & $\begin{array}{r}19-25 \text { years } \\
21.2 \pm 1.2 \\
\end{array}$ & CT & $\begin{array}{l}\text { Silicosis may cause immediate } \\
\text { mortality, especially in young } \\
\text { people. MDCT can play an } \\
\text { important role in the early } \\
\text { detection of silicosis. }\end{array}$ & Turkey \\
\hline Ehrlich et al. [42] & $\begin{array}{l}\text { Estimate the effect of respirable } \\
\text { dust and quartz exposure on } \\
\text { spirometric lung function. }\end{array}$ & Cross-sectional & $\begin{array}{c}\begin{array}{c}\text { Black South } \\
\text { African gold } \\
\text { miners }\end{array} \\
6.3-34.5 \text { years } \\
\begin{array}{c}\text { Mean }=21.8 \\
\text { years }\end{array} \\
\end{array}$ & $\begin{array}{l}520 \text { mine } \\
\text { workers }\end{array}$ & Not reported & $\begin{array}{c}\text { 37-60 years } \\
\begin{array}{c}\text { Mean }=46.7 \\
\text { years }\end{array} \\
\end{array}$ & $\begin{array}{l}\text { Questionnaires, } \\
\text { X-ray, spirometry }\end{array}$ & $\begin{array}{l}\text { Study demonstrated significant } \\
\text { lung function loss attributable to } \\
\text { dust exposure, mediated by } \\
\text { silicosis, pulmonary TB and/or } \\
\text { an independent dust effect. }\end{array}$ & South Africa \\
\hline
\end{tabular}


Table 2. Cont.

\begin{tabular}{|c|c|c|c|c|c|c|c|c|c|}
\hline Author (Year) & Objective/s & $\begin{array}{c}\text { Type of Study } \\
\text { (Cross-Sectional, } \\
\text { Clinical Trial, } \\
\text { Longitudinal, } \\
\text { Review) }\end{array}$ & $\begin{array}{c}\text { Population/ } \\
\text { Exposure (Years) }\end{array}$ & Population Size & Gender & Age (Years) & $\begin{array}{l}\text { Respiratory } \\
\text { Surveillance } \\
\text { Method/S Used }\end{array}$ & Outcomes & Location \\
\hline \multirow[t]{2}{*}{ Greabu et al. [43] } & \multirow{2}{*}{$\begin{array}{l}\text { Evaluate the relationships } \\
\text { between occupational exposure } \\
\text { to mine dust and salivary } \\
\text { antioxidants, blood uric acid } \\
\text { and the possible implications for } \\
\text { the causes of diseases caused by } \\
\text { exposure. }\end{array}$} & \multirow[t]{2}{*}{ Cross-sectional } & \multirow{2}{*}{$\begin{array}{l}\text { Long-term } \\
\text { occupational } \\
\text { exposure in } \\
\text { non-ferrous } \\
\text { metal mines }\end{array}$} & $\begin{array}{l}\text { Exposed } \\
\text { workers: } 30\end{array}$ & \multirow[t]{2}{*}{ Not reported } & $\begin{array}{l}\text { Exposed: } 44.3 \\
\text { (SD) } 4.5\end{array}$ & \multirow[t]{2}{*}{ Saliva samples } & \multirow{2}{*}{$\begin{array}{l}\text { First study to describe saliva and } \\
\text { serum parameters involved in } \\
\text { antioxidant protection and } \\
\text { metabolic regulations in } \\
\text { non-ferrous metal miners. }\end{array}$} & \multirow[t]{2}{*}{ Romania } \\
\hline & & & & Control: 30 & & $\begin{array}{l}\text { Control: } 51.3 \\
\text { (SD) } 5.6\end{array}$ & & & \\
\hline \multirow[t]{2}{*}{ Guo et al. [44] } & \multirow{2}{*}{$\begin{array}{c}\text { Survey and identify } \\
\text { differentially expressed } \\
\text { circulating miRNAs by miRNA } \\
\text { deep sequencing blood samples } \\
\text { from patients at varying stages } \\
\text { of CWP. }\end{array}$} & \multirow[t]{2}{*}{ Case-control } & \multirow[t]{2}{*}{$\mathrm{N} / \mathrm{A}$} & Cases: 30 & \multirow[t]{2}{*}{$\mathrm{N} / \mathrm{A}$} & \multirow[t]{2}{*}{$\mathrm{N} / \mathrm{A}$} & \multirow[t]{2}{*}{ Blood samples } & $\begin{array}{l}\text { Demonstrated that expressed } \\
\text { circulating miRNAs showed } \\
\text { dynamic expression patterns } \\
\text { across diseased samples. This } \\
\text { suggests these miRNAs may have }\end{array}$ & \multirow[t]{2}{*}{ China } \\
\hline & & & & $\begin{array}{c}\text { Controls: } 10 \\
n=456\end{array}$ & & & & $\begin{array}{l}\text { critical roles in the occurrence and } \\
\text { development of CWP. }\end{array}$ & \\
\hline Johnsen et al. [45] & $\begin{array}{l}\text { Investigate the relationship } \\
\text { between dust exposure and } \\
\text { annual change in lung function } \\
\text { among Norwegian silicon } \\
\text { carbide exposed workers using a } \\
\text { quantitative job matrix (JEM) } \\
\text { regarding total dust }\end{array}$ & $\begin{array}{l}\text { Longitudinal } \\
\text { cohort }\end{array}$ & $\begin{array}{l}\text { Workers in } \\
\text { Norwegian } \\
\text { silicon carbide } \\
\text { plants }\end{array}$ & $\begin{array}{l}\text { See Table S2a,b. } \\
\text { Examinations = } \\
1499\end{array}$ & $\mathrm{~N} / \mathrm{A}$ & $\mathrm{N} / \mathrm{A}$ & $\begin{array}{l}\text { Questionnaires, } \\
\text { spirometry, JEM } \\
\text { (dust exposure } \\
\text { matrix) }\end{array}$ & $\begin{array}{l}\text { Dust exposure, expressed by } \\
\text { quantitative JEM, was found to be } \\
\text { associated with an increased yearly } \\
\text { decline in FEV1. A dose-response } \\
\text { relationship was found. }\end{array}$ & Norway \\
\hline $\begin{array}{c}\text { Kahraman et al. } \\
{[46]}\end{array}$ & $\begin{array}{l}\text { Document pulmonary function } \\
\text { and prevalence of } \\
\text { pneumoconiosis in dental } \\
\text { prosthetic technicians }\end{array}$ & Cross-sectional & $\begin{array}{c}\text { Dental prosthetic } \\
\text { technicians } \\
16.7 \pm 8.4(4-43)\end{array}$ & $n=76$ & Male & $32 \pm 8,(18-55)$ & $\begin{array}{l}\text { Physical } \\
\text { examination, } \\
\text { Pulmonary } \\
\text { function test, } \\
\text { HRCT }\end{array}$ & $\begin{array}{l}\text { First prevalence study in dental } \\
\text { prosthetic technicians using HRCT. } \\
\text { Pneumoconiosis was detected in } \\
46 \% \text {, possible because HRCT is } \\
\text { able to detect very early changes. }\end{array}$ & Turkey \\
\hline $\begin{array}{l}\text { Kamaludin et al. } \\
{[47]}\end{array}$ & $\begin{array}{l}\text { Determine biomarker to be used } \\
\text { in diagnosis of occupational } \\
\text { airways inflammation from } \\
\text { occupational inorganic dust } \\
\text { exposure. }\end{array}$ & Review & N/A & N/A & N/A & $\mathrm{N} / \mathrm{A}$ & Biomarkers & Three biomarkers were identified. & Malaysia \\
\hline Larici et al. [48] & $\begin{array}{l}\text { Highlight the current role of } \\
\text { imaging, describe classic as well } \\
\text { as uncommon HRCT patterns } \\
\text { helpful in guiding diagnosis. }\end{array}$ & Review & N/A & N/A & $\mathrm{N} / \mathrm{A}$ & $\mathrm{N} / \mathrm{A}$ & HRCT & $\begin{array}{l}\text { HRCT is the best imaging modality. } \\
\text { Imaging plays a role in diagnosis, } \\
\text { surveillance, and prediction. }\end{array}$ & N/A \\
\hline
\end{tabular}


Table 2. Cont.

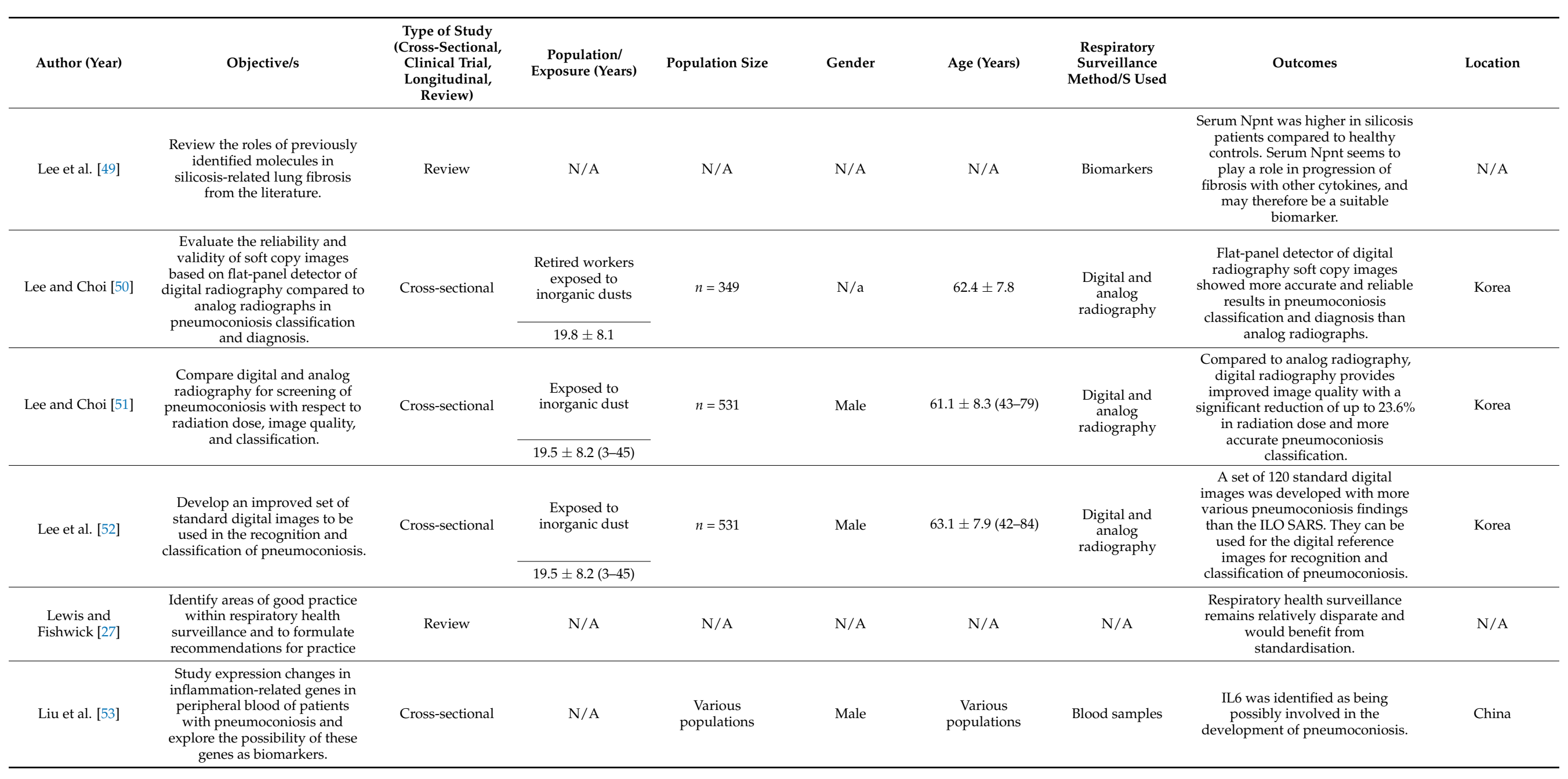


Table 2. Cont.

\begin{tabular}{|c|c|c|c|c|c|c|c|c|c|}
\hline Author (Year) & Objective/s & $\begin{array}{c}\text { Type of Study } \\
\text { (Cross-Sectional, } \\
\text { Clinical Trial, } \\
\text { Longitudinal, } \\
\text { Review) }\end{array}$ & $\begin{array}{c}\text { Population/ } \\
\text { Exposure (Years) }\end{array}$ & Population Size & Gender & Age (Years) & $\begin{array}{c}\text { Respiratory } \\
\text { Surveillance } \\
\text { Method/S Used }\end{array}$ & Outcomes & Location \\
\hline McBean et al. [6] & $\begin{array}{l}\text { Understand the radiological } \\
\text { presentation of individuals } \\
\text { diagnosed with coal mine dust } \\
\text { lung disease since } 2015 \text { in } \\
\text { Queensland. }\end{array}$ & Case series & $\begin{array}{c}\text { Individuals } \\
\text { identified as } \\
\text { having coal mine } \\
\text { dust lung disease } \\
\text { (CMDLD) since } \\
2015 \\
\begin{array}{c}\text { Mean: } 26.2 \text { years } \\
\text { (range: 6-45) }\end{array}\end{array}$ & 79 & Male & $\begin{array}{c}\text { Mean }=58.9 \\
\text { years (range: } \\
\text { 35-90) }\end{array}$ & $\begin{array}{c}\text { Questionnaires, } \\
\text { X-ray and/or CT, } \\
\text { spirometry }\end{array}$ & $\begin{array}{l}\text { First study in over } 30 \text { years to } \\
\text { investigate the radiological } \\
\text { presentation of CMDLD in QLD, } \\
\text { and the first ever to incorporate } \\
\text { HRCT. Approximately } 30 \% \text { of } \\
\text { subjects had advanced disease. } \\
\text { Findings of interest included the } \\
\text { high burden of opacities observed } \\
\text { and the presence of RCS-related } \\
\text { features in the majority of subjects. }\end{array}$ & Australia \\
\hline Miao et al. [55] & $\begin{array}{l}\text { Conduct proteomic profiling for } \\
\text { the early stages of silicosis to } \\
\text { investigate the pathophysiology } \\
\text { and to identify potential } \\
\text { candidate proteins for early } \\
\text { diagnosis. }\end{array}$ & Case-control & $\begin{array}{c}\text { Dust-exposed } \\
\text { workers without } \\
\text { silicosis; silicosis } \\
\text { patients; Healthy } \\
\text { controls }\end{array}$ & 45 & N/A & $55-64$ & $\begin{array}{l}\text { X-ray, blood } \\
\text { sample }\end{array}$ & $\begin{array}{l}\text { A number of proteins involved in } \\
\text { silicosis development were } \\
\text { identified, with a large number of } \\
\text { proteins and peptides being } \\
\text { dramatically altered during early } \\
\text { development. This may contribute } \\
\text { to future work to identify potential } \\
\text { biomarkers. }\end{array}$ & China \\
\hline \multirow{3}{*}{ Nardi et al. [56] } & \multirow{3}{*}{$\begin{array}{l}\text { Evaluate inflammatory and } \\
\text { oxidative stress parameters as } \\
\text { potential early biomarkers for } \\
\text { RCS exposure. }\end{array}$} & \multirow{3}{*}{ Case-control } & \multirow{3}{*}{$\begin{array}{l}\text { CS exposed } \\
\text { miners }\end{array}$} & $\begin{array}{c}\text { Workers exposed } \\
\text { to } C S=38\end{array}$ & \multirow{3}{*}{ Male } & \multirow{3}{*}{$\begin{array}{c}\text { Various, see } \\
\text { Table } 1 \text { in article }\end{array}$} & \multirow{3}{*}{$\begin{array}{l}\text { Blood sample, } \\
\text { anthropometric } \\
\text { measurements, }\end{array}$} & \multirow{3}{*}{$\begin{array}{l}\text { For the first time, this study } \\
\text { suggested L-selectin surface } \\
\text { protein expression in lymphocytes } \\
\text { might be a potential biomarker for } \\
\text { monitoring CS toxicity in workers } \\
\text { with at least } 16 \text { years exposure. }\end{array}$} & \multirow{3}{*}{ Brazil } \\
\hline & & & & $\begin{array}{c}\text { With silicosis = } \\
24\end{array}$ & & & & & \\
\hline & & & & $\begin{array}{c}\text { Unexposed } \\
\text { workers }=30\end{array}$ & & & & & \\
\hline $\begin{array}{c}\text { Okumura et al. } \\
{[57]}\end{array}$ & $\begin{array}{l}\text { Investigate the effects of } \\
\text { parameters on overall } \\
\text { classification performance. } \\
\text { Develop enhancement methods } \\
\text { to reduce false-positive and } \\
\text { false-negative values in a CAD } \\
\text { scheme for pneumoconiosis. }\end{array}$ & $\begin{array}{l}\text { Retrospective, } \\
\text { cross-sectional }\end{array}$ & N/A & N/A & N/A & N/A & $\begin{array}{l}\text { Chest } \\
\text { radiographs }\end{array}$ & $\begin{array}{l}\text { Successfully developed a CAD } \\
\text { system using three new } \\
\text { enhancement methods for } \\
\text { classification of pneumoconiosis } \\
\text { chest radiographs. }\end{array}$ & Japan \\
\hline
\end{tabular}


Table 2. Cont.

\begin{tabular}{|c|c|c|c|c|c|c|c|c|c|}
\hline Author (Year) & Objective/s & $\begin{array}{c}\text { Type of Study } \\
\text { (Cross-Sectional, } \\
\text { Clinical Trial, } \\
\text { Longitudinal, } \\
\text { Review) }\end{array}$ & $\begin{array}{c}\text { Population/ } \\
\text { Exposure (Years) }\end{array}$ & Population Size & Gender & Age (Years) & $\begin{array}{l}\text { Respiratory } \\
\text { Surveillance } \\
\text { Method/S Used }\end{array}$ & Outcomes & Location \\
\hline \multirow[t]{2}{*}{ Ophir et al. [58] } & \multirow[t]{2}{*}{$\begin{array}{l}\text { Screen exposed workers using } \\
\text { quantitative biometric } \\
\text { monitoring of functional and } \\
\text { inflammatory parameters. }\end{array}$} & \multirow[t]{2}{*}{ Case-control } & $\begin{array}{l}\text { Artificial stone } \\
\text { workers }\end{array}$ & $\begin{array}{c}\text { Exposed } \\
\text { workers: } 68\end{array}$ & \multirow[t]{2}{*}{ Male } & $\begin{array}{c}\text { Exposed workers: } \\
48.6 \pm 11.4\end{array}$ & \multirow[t]{2}{*}{$\begin{array}{l}\text { Questionnaires, } \\
\text { PFT, induced } \\
\text { sputum }\end{array}$} & \multirow{2}{*}{$\begin{array}{l}\text { Reports first application of XRF } \\
\text { technology for quantifying } \\
\text { elements in biological samples. } \\
\text { PFT were significantly lower for } \\
\text { exposed workers. Also IS in } \\
\text { exposed workers showed } \\
\text { significantly higher neutrophilic } \\
\text { inflammation. Particle size in IS of } \\
\text { exposed workers was similar to } \\
\text { the artificial stone dust. }\end{array}$} & \multirow[t]{2}{*}{ Israel } \\
\hline & & & Up to 20 years & Controls: 48 & & $\begin{array}{c}\text { Controls: } \\
38.0 \pm 17.1\end{array}$ & & & \\
\hline \multirow[t]{2}{*}{$\begin{array}{c}\text { Palabiyik et al. } \\
\text { [12] }\end{array}$} & \multirow{2}{*}{$\begin{array}{l}\text { Investigate if occupational silica } \\
\text { exposure results in alterations in } \\
\text { neopterin levels, tryptophan } \\
\text { degradation, and activities of } \\
\text { superoxide dismutase and } \\
\text { catalase, agents in the } \\
\text { antioxidant defense system. }\end{array}$} & \multirow[t]{2}{*}{ Case-control } & $\begin{array}{l}\text { Denim } \\
\text { sandblasting } \\
\text { workers }\end{array}$ & $\begin{array}{l}\text { Silicosis patients: } \\
55\end{array}$ & \multirow[t]{2}{*}{ Male } & $\begin{array}{l}\text { Silicosis patients: } \\
30 \pm 1(21-48)\end{array}$ & \multirow{2}{*}{$\begin{array}{l}\text { Questionnaires, } \\
\text { PFT, blood } \\
\text { samples }\end{array}$} & \multirow{2}{*}{$\begin{array}{l}\text { Denim sandblasters exposed to } \\
\text { silica had increased neopterin } \\
\text { levels and tryptophan degradation } \\
\text { confirming the possibility of their } \\
\text { use as indicators of cellular } \\
\text { immune response. }\end{array}$} & \multirow[t]{2}{*}{ Turkey } \\
\hline & & & $\begin{array}{l}33.6 \pm 23.8(2 \text { to } \\
120) \text { months }\end{array}$ & Controls: 22 & & $\begin{array}{c}\text { Controls: } 36 \pm 10 \\
(18-52)\end{array}$ & & & \\
\hline \multirow{3}{*}{ Pelclová et al. [59] } & \multirow{3}{*}{$\begin{array}{l}\text { Evaluate the potential impact of } \\
\text { lung fibrosis on the levels of } \\
\text { oxidative stress markers in } \\
\text { blood and urine of workers } \\
\text { exposed to silica. }\end{array}$} & \multirow{3}{*}{ Case-control } & \multirow{3}{*}{$\begin{array}{l}\text { Various } \\
\text { occupations with } \\
\text { exposure }\end{array}$} & $\begin{array}{c}\text { Asbestos } \\
\text { exposed workers: } \\
45\end{array}$ & $\begin{array}{c}\text { Asbestos } \\
\text { exposed workers: } \\
24 \text { male }\end{array}$ & $\begin{array}{c}\text { Asbestos } \\
\text { exposed workers: } \\
69.6 \pm 2.0\end{array}$ & \multirow{3}{*}{$\begin{array}{l}\text { Questionnaires, } \\
\text { physical } \\
\text { examination, } \\
\text { X-ray, CT, blood } \\
\text { sample, urine } \\
\text { sample, lung } \\
\text { function, EBC }\end{array}$} & \multirow{3}{*}{$\begin{array}{l}\text { 8-isoprostane appears to be the } \\
\text { optimal oxidative stress marker for } \\
\text { respiratory disorders. HNE can be } \\
\text { used as a marker for } \\
\text { pneumoconiosis. Findings support } \\
\text { the suggestion that EBC can } \\
\text { contribute to a better } \\
\text { understanding of the pathogenesis } \\
\text { of silicosis. }\end{array}$} & \multirow{3}{*}{ Czech Republic } \\
\hline & & & & $\begin{array}{l}\text { Silica exposed } \\
\text { workers: } 37\end{array}$ & $\begin{array}{l}\text { Silica exposed } \\
\text { workers: } 36 \mathrm{male}\end{array}$ & $\begin{array}{l}\text { Silica exposed } \\
\text { workers: } \\
69.1 \pm 2.9\end{array}$ & & & \\
\hline & & & & Controls: 29 & Controls: 20 male & $\begin{array}{l}\text { Controls: } \\
67.0 \pm 4.6\end{array}$ & & & \\
\hline \multirow{3}{*}{ Pelclová et al. [60] } & \multirow{3}{*}{$\begin{array}{l}\text { Measure multiple markers in the } \\
\text { EBC, plasma and urine of } \\
\text { exposed workers to determine } \\
\text { the possible impact of systemic } \\
\text { disease, pharmaceuticals and } \\
\text { diet on EBC levels. }\end{array}$} & \multirow{3}{*}{ Case-control } & \multirow{3}{*}{$\begin{array}{l}\text { Various } \\
\text { occupations with } \\
\text { exposure }\end{array}$} & $\begin{array}{c}\text { Asbestos } \\
\text { exposed workers: } \\
45\end{array}$ & $\begin{array}{l}\text { Asbestos } \\
\text { exposed workers: } \\
24 \text { male }\end{array}$ & $\begin{array}{l}\text { Asbestos } \\
\text { exposed workers: } \\
69.6 \pm 2.0\end{array}$ & \multirow{3}{*}{$\begin{array}{l}\text { Questionnaires, } \\
\text { physical } \\
\text { examination, } \\
\text { X-ray, CT, blood } \\
\text { sample, urine } \\
\text { sample, EBC }\end{array}$} & \multirow{3}{*}{$\begin{array}{l}\text { Findings suggest that for the } \\
\text { detection of pneumoconiosis EBC } \\
\text { is the most useful compared to } \\
\text { plasma and urine. }\end{array}$} & \multirow{3}{*}{ Czech Republic } \\
\hline & & & & $\begin{array}{l}\text { Silica exposed } \\
\text { workers: } 37\end{array}$ & $\begin{array}{l}\text { Silica exposed } \\
\text { workers: } 36 \text { male }\end{array}$ & $\begin{array}{l}\text { Silica exposed } \\
\text { workers: } \\
69.1 \pm 2.9 \\
\end{array}$ & & & \\
\hline & & & & Controls: 27 & Controls: 18 male & $\begin{array}{l}\text { Controls: } \\
66.0 \pm 6.9\end{array}$ & & & \\
\hline Sato et al. [61] & $\begin{array}{l}\text { Identify predictive factors of } \\
\text { excess decline in FEV1 in } \\
\text { patients with chronic silicosis. }\end{array}$ & Cross-sectional & $\begin{array}{c}\text { Exposed workers } \\
21.9 \pm 12.3\end{array}$ & $n=33$ & Male & $73.5 \pm 5.7$ & $\begin{array}{l}\text { Questionnaires, } \\
\text { X-ray, spirometry, } \\
\text { blood samples }\end{array}$ & $\begin{array}{c}\text { Serum H01 may be a useful } \\
\text { marker of lung function decline } \\
\text { and disease progression in silicosis } \\
\text { patients. }\end{array}$ & Japan \\
\hline
\end{tabular}


Table 2. Cont.

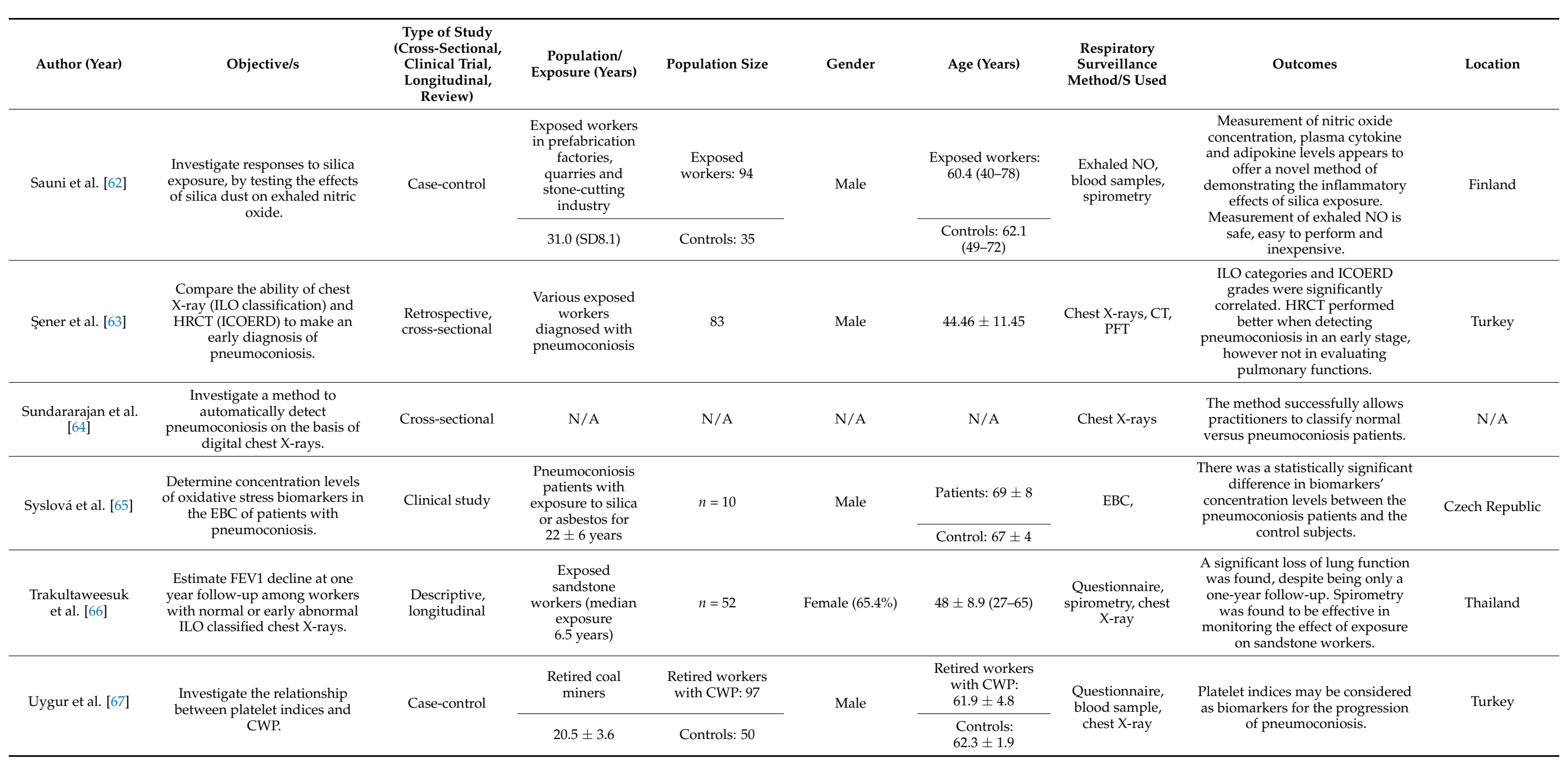


Table 2. Cont.




Table 2. Cont.

\begin{tabular}{|c|c|c|c|c|c|c|c|c|c|}
\hline Author (Year) & Objective/s & $\begin{array}{l}\text { Type of Study } \\
\text { (Cross-Sectional, } \\
\text { Clinical Trial, } \\
\text { Longitudinal, } \\
\text { Review) }\end{array}$ & $\begin{array}{c}\text { Population/ } \\
\text { Exposure (Years) }\end{array}$ & Population Size & Gender & Age (Years) & $\begin{array}{l}\text { Respiratory } \\
\text { Surveillance } \\
\text { Method/S Used }\end{array}$ & Outcomes & Location \\
\hline Young et al. [72] & $\begin{array}{l}\text { Evaluate the use of CAD to } \\
\text { diagnose both TB and silicosis in } \\
\text { a population with a high burden } \\
\text { of both diseases. }\end{array}$ & Quantitative & N/A & N/A & N/A & N/A & X-ray & $\begin{array}{l}\text { Using CAD as a mass screening } \\
\text { tool for TB and silicosis shows } \\
\text { promise, however current ability } \\
\text { to differentiate between the two is } \\
\text { limited. The successful use of CAD } \\
\text { to streamine the process of } \\
\text { detection requires knowledge of } \\
\text { the local context. }\end{array}$ & South Africa \\
\hline Yu et al. [73] & $\begin{array}{l}\text { Establish an automated scheme } \\
\text { for CAD of pneumoconiosis in } \\
\text { X-rays }\end{array}$ & Quantitative & $\mathrm{N} / \mathrm{A}$ & $\begin{array}{l}\text { Pneumoconiosis: } \\
125\end{array}$ & N/A & N/A & X-ray & $\begin{array}{l}\text { Findings show high classification } \\
\text { performances. The fully } \\
\text { automated scheme developed in } \\
\text { this study has a higher accuracy } \\
\text { and a more convenient interaction } \\
\text { compared to previous methods. } \\
\text { Scheme may be helpful to } \\
\text { clinicians using CAD for mass } \\
\text { chest screening and interpreting } \\
\text { and differentiating between } \\
\text { normal and pneumoconiosis cases. }\end{array}$ & China \\
\hline \multirow[t]{3}{*}{ Zhang et al. [74] } & \multirow{3}{*}{$\begin{array}{l}\text { Construct a phage display } \\
\text { human antibody library } \\
\text { (PDHAL) against } \\
\text { pneumoconiosis for the } \\
\text { diagnosis and treatment of CWP. }\end{array}$} & \multirow[t]{3}{*}{ Case-control } & \multirow[t]{3}{*}{$\mathrm{N} / \mathrm{A}$} & $\begin{array}{c}\text { Coal workers } \\
\text { with CWP: } 558 \\
\end{array}$ & \multirow[t]{3}{*}{ Male } & $\begin{array}{c}\text { Controls: } \\
36.88 \pm 9.39 \\
\end{array}$ & \multirow[t]{3}{*}{$\begin{array}{l}\text { Blood samples, } \\
\text { BALF }\end{array}$} & \multirow{3}{*}{$\begin{array}{l}\text { A PDHAL against CWP was } \\
\text { established and six strong positive } \\
\text { clones were selected, sequenced } \\
\text { and identified. Protective factors } \\
\text { were identified. Serum and } \\
\text { antibodies that could be used as } \\
\text { potential biomarkers for the } \\
\text { diagnosis and treatment of CWP } \\
\text { were identified. }\end{array}$} & \multirow[t]{3}{*}{ China } \\
\hline & & & & $\begin{array}{l}\text { Coal workers } \\
\text { without CWP: } \\
309 \\
\end{array}$ & & & & & \\
\hline & & & & Control: 393 & & & & & \\
\hline Zhao et al. [75] & $\begin{array}{l}\text { Describe a CAD method to } \\
\text { classify pneumoconiosis on } \\
\text { HRCT images. }\end{array}$ & Quantitative & N/A & $\begin{array}{c}\text { Subjects:112 } \\
\text { HRCT scans: } 175\end{array}$ & N/A & $\mathrm{N} / \mathrm{A}$ & HRCT & $\begin{array}{l}\text { Findings indicate that the method } \\
\text { developed could be helpful in } \\
\text { classifying pneumoconiosis on } \\
\text { HRCT. }\end{array}$ & Japan \\
\hline Zhu et al. [76] & $\begin{array}{l}\text { Propose a multi-scale opacity } \\
\text { detection approach to detect } \\
\text { suspected opacities from X-ray }\end{array}$ & Quantitative & N/A & $\mathrm{N} / \mathrm{A}$ & N/A & N/A & X-ray & $\begin{array}{l}\text { Findings demonstrate the } \\
\text { approach to be effective in } \\
\text { detecting and recognising silicosis } \\
\text { opacity. The approach successfully } \\
\text { revealed changes in silicosis } \\
\text { pathology and may be adopted as } \\
\text { an appropriate tool for automatic } \\
\text { silicosis diagnosis. }\end{array}$ & China \\
\hline
\end{tabular}


In addition, following the search methods outlined above, there were 19 grey literature sources screened and included in the final review; these are summarised in Table 3.

Table 3. List of grey literature included in the review.

\begin{tabular}{|c|c|}
\hline Author/Organisation & Available at \\
\hline Alif et al. [1] & $\begin{array}{c}\text { https://www.safeworkaustralia.gov.au/doc/occupational-lung-diseases- } \\
\text { australia-2006-2019 }\end{array}$ \\
\hline International Labour Organisation (ILO) & $\begin{array}{l}\text { https://www.ilo.org/global/topics/safety-and-health-at-work/ } \\
\text { areasofwork/occupational-health/WCMS_108548/lang--en/index.htm }\end{array}$ \\
\hline $\begin{array}{l}\text { Australian and New Zealand Society of } \\
\text { Occupational Medicine (ANZSOM) }\end{array}$ & https://www.anzsom.org.au/ \\
\hline Coal Services NSW & $\begin{array}{l}\text { https:/ /www.coalservices.com.au/wp-content/uploads/2019/11/2018 } \\
\text { 0625_Order-43_Information-for-employers_updated-Nov2019.pdf }\end{array}$ \\
\hline Icare & $\begin{array}{c}\text { https://www.icare.nsw.gov.au/news-and-stories/reducing-worker- } \\
\text { risks-for-silica }\end{array}$ \\
\hline Lung Foundation Australia & https://lungfoundation.com.au/drive-change/ \\
\hline $\begin{array}{c}\text { National Institute for Occupational Safety and } \\
\text { Health (NIOSH) }\end{array}$ & $\begin{array}{l}\text { https://www.cdc.gov/niosh/topics/silica/default.html } \\
\text { https:// www.cdc.gov/niosh/topics / surveillance/ORDS/ } \\
\text { https:/ / www.cdc.gov/niosh/topics/surveillance/ords/ } \\
\text { workermedicalmonitoring.html } \\
\text { https:/ / www.cdc.gov/niosh/docs/2005-110/nmed0205.html; } \\
\text { https:/ / www.cdc.gov/niosh/docs/81-123/default.html }\end{array}$ \\
\hline $\begin{array}{c}\text { Royal Australian and New Zealand College of } \\
\text { Radiologists (RANZCR) }\end{array}$ & https://www.ranzcr.com/search/silicosis-position-statement \\
\hline Royal Australian College of Physicians & $\begin{array}{c}\text { https:// www.racp.edu.au/advocacy/division-faculty-and-chapter- } \\
\text { priorities / faculty-of-occupational-environmental-medicine/accelerated- } \\
\text { silicosis/faqs }\end{array}$ \\
\hline Safe Work Australia & https://www.safeworkaustralia.gov.au/silica \\
\hline SafeWork NSW & $\begin{array}{l}\text { https://www.safework.nsw.gov.au/hazards-a-z/hazardous-chemical/ } \\
\text { priority-chemicals/crystalline-silica }\end{array}$ \\
\hline SafeWork Qld & https://www.worksafe.qld.gov.au/silicosis/background-to-silicosis \\
\hline Thoracic Society of Australia and New Zealand & $\begin{array}{c}\text { https://www.thoracic.org.au/respiratorylaboratoryaccreditation/ } \\
\text { spirometry-standards }\end{array}$ \\
\hline TSANZ & https://www.thoracic.org.au/documents/item/407 \\
\hline WorkCover WA & https://www.workcover.wa.gov.au/workers/silicosis-claims/ \\
\hline WorkSafe ACT & $\begin{array}{l}\text { https://www.accesscanberra.act.gov.au/app/answers/detail/a_id/4646 } \\
\qquad / \sim / \text { silica-dust }\end{array}$ \\
\hline WorkSafe NZ & $\begin{array}{l}\text { https://worksafe.govt.nz/topic-and-industry/dust/silica-dust-in-the- } \\
\text { workplace/ }\end{array}$ \\
\hline WorkSafe Tasmania & https://worksafe.tas.gov.au/silicasafe \\
\hline WorkSafe Victoria & https://www.worksafe.vic.gov.au/crystalline-silica \\
\hline
\end{tabular}

The countries represented by the peer-reviewed literature included in the scoping review are shown in Figure 2.

The worldwide occurrence of silicosis was demonstrated in the spatial distribution of the studies included. This was not necessarily an exhaustive list of all countries that had incidences of silicosis.

Many studies included in this review reported a high incidence of smoking among participants. This confounder makes it difficult to isolate the impacts of silica dust exposure from the damage caused by smoking. It is common practice for cessation of smoking 
programs to be promoted at screening appointments and to participants in silicosis research studies.

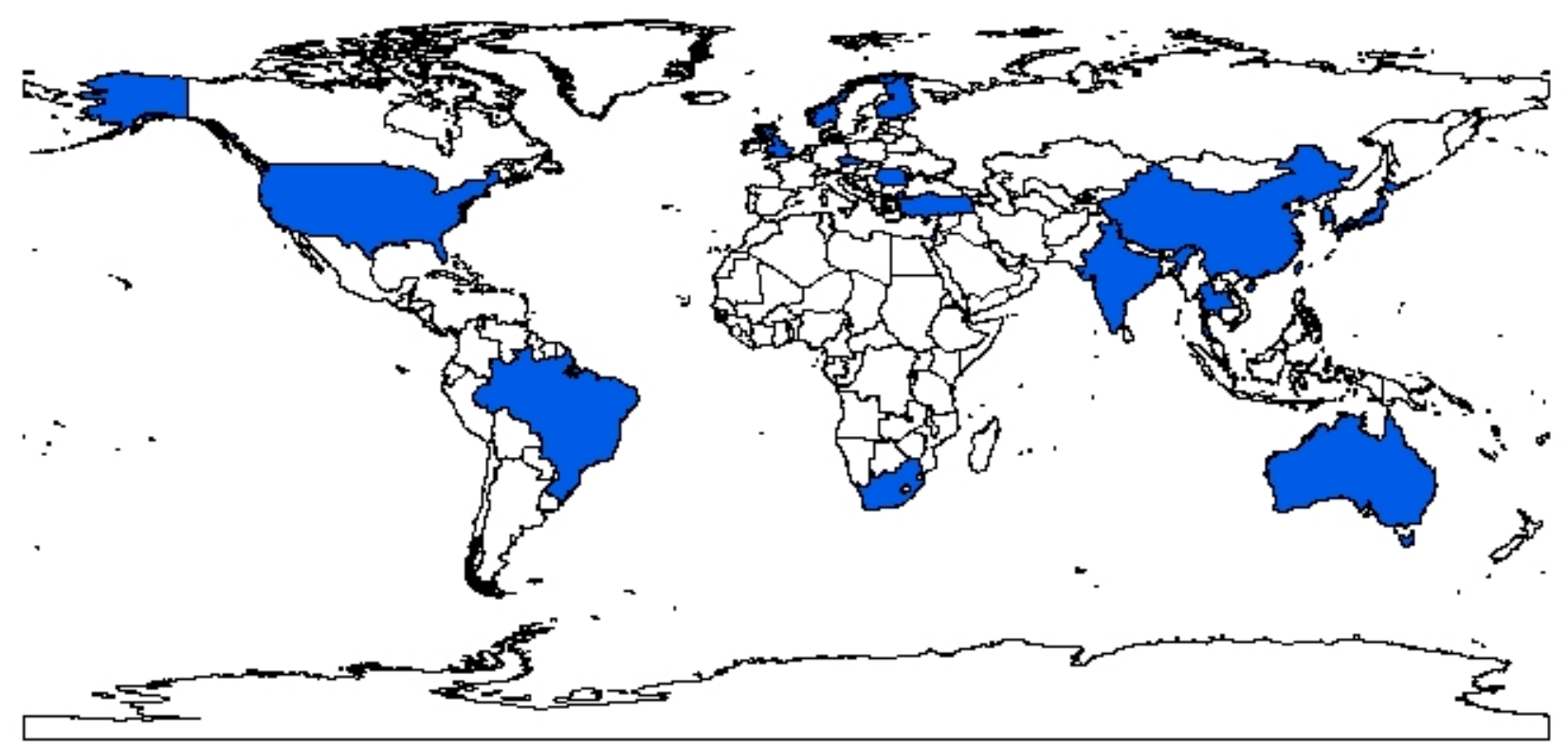

Countries represented in the articles included in the scoping review

Figure 2. Global incidence of silicosis in the journal articles included in the scoping review.

\section{Clinical Trials}

OpenTrials returned 44 entries when we searched for "silicosis" and "pneumoconiosis", however, these trials either had no results available or were testing drugs for treatment. It appears from the search conducted for this review that clinical trials investigating early detection methods for silicosis are rare.

\section{Discussion}

This review was guided by the overarching aim to inform changes in respiratory surveillance with the global goal to reduce the prevalence of silicosis and improve the prognosis of workers who develop silicosis. Some articles included in the scoping review focus more broadly on pneumoconiosis in general or other types of pneumoconiosis, such as CWP. The methods investigated in these articles are pertinent to respiratory surveillance for silicosis. Different surveillance methods were identified, including spirometry, imaging, and HRCT, and these are discussed in more detail below.

\subsection{Spirometry}

Spirometry is a type of pulmonary function test. Spirometry is currently used for diagnosing the risk of damage, identifying lung disease, monitoring workers exposed to particulate matter, and to evaluate therapeutic interventions [35]. Although spirometry has been used as the first-choice method to evaluate pulmonary alterations in workers exposed to particulate matter, spirometry has limited sensitivity when detecting abnormalities before extensive damage occurs [35]. In addition, there are different standards for the procedure itself, for example, in Australia, the test must be performed for coal mine workers by practitioners with a particular qualification [77], but this is not required in other occupations.

Spirometry, or some form of pulmonary function test, was used in many of the studies in the scoping review. In these investigations, spirometry was always accompanied by health and exposure questionnaires and, in most cases, by additional surveillance methods, such as chest X-ray or HRCT $[20,45,61,62,66]$. Spirometry can contribute to the diagnosis 
and monitoring of pneumoconiosis, and specifically, Trakultaweesuk et al. [66] found that spirometry, using a mean decline in $\mathrm{FEV}_{1}$ of $272.0 \pm 155.5$, was a good parameter for the respiratory surveillance of silica-exposed workers. It is important to note that spirometry and questionnaires alone are not able to diagnose the difference between silicosis and COPD. Despite the widespread use of spirometry, the practical implications and inconsistencies in performing the test must be considered [77].

\subsection{Imaging}

Respiratory surveillance routinely incorporates imaging, including chest radiography (X-ray) and/or HRCT. Globally, it is typical for chest X-rays to be assessed according to the International Labour Organisation (ILO) Classification System [78]. In addition, many jurisdictions have the requirement for an NIOSH B Reader to assess the chest X-ray, a certification granted to physicians who demonstrate proficiency in the classification of chest X-rays for pneumoconioses using the ILO Classification System [15].

It has been identified that chest $X$-rays are failing to reliably detect occupational lung disease $[19,79]$. For example, in a cohort of workers from Queensland, $43 \%$ had chest $\mathrm{X}$-rays classified as normal using the ILO Classification System, however, the disease was visible on HRCT [19]. Non-occupational lung disease is now diagnosed using CT, and it is recommended that HRCT also replace chest $\mathrm{X}$-ray for the diagnosis of occupational lung disease due to CT's higher sensitivity to detect early disease and greater accuracy in characterising patterns of disease $[19,80,81]$. The Royal Australian and New Zealand College of Radiologists [19] strongly recommend CT as the primary imaging modality to be used for respiratory surveillance in exposed workers. This recommendation is supported by Kahraman et al. [46], and the references therein.

Specifically, HRCT has an enhanced capacity to detect pneumoconiosis compared to chest $\mathrm{X}$-ray due to the increased sensitivity provided by the finer spatial resolution and 3D nature of HRCT [6]. While Larici et al. [48] concluded that HRCT is the optimal modality of imaging, Şener et al. [63] resolved that, although HRCT had a higher rate of detection in the early stages, the cost, radiation exposure, accessibility, and lack of ability to evaluate pulmonary functions did not support the introduction of routine use in this setting.

In some jurisdictions, in this case Korea, analog radiography persists as the standard for respiratory surveillance. Lee and Choi [50] concluded that soft images from a flat-panel detector of digital radiography provide more accurate and reliable results in pneumoconiosis classification and diagnosis than analog radiographs, and concluded that, in the circumstance where HRCT is not available, digital radiograph is preferred. Conflictingly, [69] found digital and analog radiography to be equivalent.

There is a body of work that investigates the automatic classification of chest Xrays $[57,64,72,73,75,76]$. In some locations, there is a lack of expertise in the diagnosis of occupational lung disease, and it appears that these technologies have the capacity to assist by automatically detecting abnormalities in chest X-rays.

As stated previously, detection in the early stages of silicosis has challenges. McBean et al. [6] described radiologists as being at the frontline in occupational lung screening and that they must be aware of the imaging spectrum.

\subsection{Biomarkers}

There is a growing body of epidemiological research that focuses on validating biomarkers by assessing their ability to indicate exposure, effect, disease, or susceptibility [82]. When used in health surveillance, biomarkers can be indicators of hazard, exposure, disease, and population risk [83]. The overarching goal of using biomarkers is to provide insight into the pathogenesis of silicosis and the biological mechanisms that underpin its progression. This review identified a number of studies that aimed to validate particular biomarkers as indicators of silicosis $[4,36,37,42,49,61,67]$. The grey literature search returned several Chinese articles about biomarkers that could not be accessed. 
Thakkar et al. [84] identified that existing studies that consider biomarkers have been conducted with cross-sectional methods within a group population over a short time period. These findings give statistical and probabilistic results in terms of an individual subject. However, what is needed is an observation of biomarkers over time, i.e., a longitudinal cohort study is essential. A study of this design would have prognostic value and contribute to workers adopting preventive strategies, while also reducing individual cases of silicosis [84].

Many studies test for biomarkers of oxidative stress, an imbalance in the body between the production of free radicals and the antioxidant defense [65]. Oxidative stress can lead to damage in biological tissue as a result of an imbalance between oxidants and antioxidants [82]. Metals found in mine dust have the potential to induce oxidative stress, which can cause harmful effects to the human body [43]. The ability of a chemical to exert biological effects dictates the capacity to generate oxidative stress [43]. Oxidative stress has been identified as strongly related to the severity of silicosis [12]. However, the parameters of oxidative stress that represent silicosis remain invalidated.

There are many avenues of biomarkers that require further investigation. It has been identified that, in the search for biomarkers for pneumoconiosis, there is a need to investigate biomarkers that play important roles in screening, diagnosis [74], and disease progression [85]. In addition, Schulte [83] notes the need to justify the cost and difficulty in obtaining samples. Pandey and Agarwal [85] emphasise the need for a cohort and longitudinal study of the potential biomarkers in vulnerable groups.

A large number of biomarkers with the potential to detect lung disease were investigated in the literature summarised in this review, including (but not limited to): Club/Clara cell protein 16 (CC16) [21]; serum HO-1 [61]; IL6 [53]; TNF $\alpha$, IL6, and IL8 [47]; and Npnt [49]. It was not possible to determine a single biomarker with the most potential. Indeed, the need for research to identify biomarkers that provide insight into the pathogenesis of silicosis and the biological mechanisms that underpin its progression was abundantly clear.

\subsection{Exhaled Biomarkers}

Exhaled breath condensate (EBC) can be used to assess the respiratory health of pneumotoxic-exposed workers, as it quantifies lung tissue dose and the consequent pulmonary effects [39]. EBC is obtained by collecting exhaled cooled breath, which is analysed for volatile and non-volatile macromolecules [86]. The range of biomarkers that have been explored when investigating pneumoconiosis, including oxidative stress and inflammatoryderived biomarkers, suggests that EBC analysis may contribute to understanding the pathogenesis of the airways of exposed workers [39]. EBC analysis, as a method of studying pulmonary biomarkers of exposure, effect, and susceptibility in the workplace, proves to be one of the most promising methods currently available [39]. In particular, due to its non-invasive collection method, it is highly suitable to be applied in field studies and longitudinal assessments [39].

Although not commonly used, findings support the suggestion that EBC can contribute to an improved understanding of the pathogenesis of silicosis $[59,60]$. Indeed, when compared to plasma and urine, markers in EBC appeared to be the most useful method for detecting pneumoconiosis [60]. Leese et al. [87] demonstrated that crystalline silica particles can be detected in the EBC of exposed workers, however, there were limitations due to the volume of the sample produced.

The measurement of exhaled NO and volatile organic compounds is considered to be an inexpensive, safe, and easy-to-perform test that can be used to assess peripheral lung inflammation, and could potentially play a role in the diagnosis and follow-up of fibrosing lung disorders $[62,71]$. However, further research is needed that includes follow-up testing and investigating different levels of exposure [62].

EBC is non-invasive and highly accurate, making it an attractive option for the early detection of silicosis. Again, there is a growing body of research investigating a number of 
EBC options, and the need for further study is acknowledged [62,71,87]. Indeed, Corradi et al. [39] recognised the substantial limitations that currently exist, preventing its use as a routine method of screening in the workplace. Specifically, they identified the need for further development in the area of standardising EBC collection, data reporting, and validation of biomarkers.

\subsection{Summary of Methods}

This review identified the need to standandarise the process of respiratory surveillance. In addition, $\mathrm{X}$-ray was determined as not sufficient in detecting silicosis, while spirometry is subject to the skill and experience of the practitioner. HRCT is recognised as the optimal method, however, it is not always available. EBC and biomarkers hold promise, although, at this stage, they are not validated and remain at an investigational stage.

The strengths of this review include the search being conducted beyond peer-reviewed literature to include grey literature and supporting regulatory documentation, as well as the scoping review following the systematic PRISMA-ScR framework. However, it should be noted there are some limitations, such as including only papers published in English, and the fact that only those published since 2010 were included.

Based on the findings presented here, a number of recommendations were formulated. Firstly, there is a need for further lab- and field-based studies that monitor a range of biomarkers to successfully identify one or more biomarkers that conclusively provide insight into the pathogenesis of silicosis and the biological mechanisms that underpin its progression. Second, any future empirical studies that attempt to validate the use of biomarkers or EBC as an early detection method for silicosis must also include standard surveillance methods as a point of comparison, i.e., spirometry, X-ray, and HRCT. Lastly, future empirical studies should include a diversity of participants to allow examination of a range of scenarios, for example, diagnosed silicosis patients at different stages of the disease, exposed workers (with no previous diagnosis of silicosis, COPD, TB, fibrosis, etc.) with a varying number of years of occupational exposure, and healthy unexposed controls with no previous diagnosis of silicosis, COPD, TB, fibrosis, etc.

\section{Conclusions}

Silicosis is a debilitating and sometimes fatal disease, yet it is totally preventable. Caused by exposure to RCS, hundreds of thousands of workers worldwide are at risk of developing silicosis. The global prevalence of silicosis (and other pneumoconioses) warrants further investigation into methods for detection in the early stage of the disease. While spirometry, X-ray, and HRCT can play important roles in respiratory surveillance, there is opportunity for new methods, such as biomarkers and EBC, to become routine methods of surveillance. Any future effort to research into early detection methods for respiratory surveillance should focus on providing insight into the pathogenesis of silicosis and the biological mechanisms that underpin its progression. These efforts should include longitudinal analysis of at-risk populations.

Author Contributions: Conceptualization, C.J., J.T. and E.K.A.; methodology, C.J., J.T. and E.K.A.; formal analysis, E.K.A.; writing-original draft preparation, E.K.A.; writing-review and editing, E.K.A., C.J. and J.T. All authors have read and agreed to the published version of the manuscript.

Funding: This research was funded by Humanomics Pty Ltd., grant number G1901613, and the Department of Industry, Science, Energy and Resources (Commonwealth of Australia), grant number G2000210.

Data Availability Statement: Not applicable.

Conflicts of Interest: The authors declare no conflict of interest. 


\section{References}

1. Alif, S.M.; Glass, D.C.; Abramson, M.; Hoy, R.; Sim, M.R. Occupational Lung Diseases in Australia 2006-2019. Safe Work Australia 2020. Available online: https:/ / www.safeworkaustralia.gov.au/doc/occupational-lung-diseases-australia-2006-2019 (accessed on 7 August 2020).

2. De Vuyst, P.; Camus, P. The past and present of pneumoconioses. Curr. Opin. Pulm. Med. 2000, 6, 151-156. [CrossRef]

3. Doganay, S.; Gocmen, H.; Yikilmaz, A.; Coskun, A. Silicosis due to denim sandblasting in young people: MDCT findings. Eurasian J. Med. 2010, 41, 21-23. [CrossRef] [PubMed]

4. Chu, M.; Ji, X.; Chen, W.; Zhang, R.; Sun, C.; Wang, T.; Luo, C.; Gong, J.; Zhu, M.; Fan, J.; et al. A genome-wide association study identifies susceptibility loci of silica-related pneumoconiosis in Han Chinese. Hum. Mol. Genet. 2014, 23, 6385-6394. [CrossRef]

5. Cohen, R.A. Resurgent coal mine dust lung disease: Wave of the future or a relic of the past? Occup. Environ. Med. 2016, 73, 715-716. [CrossRef]

6. McBean, R.; Tatkovic, A.; Edwards, R.; Newbigin, K. What does coal mine dust lung disease look like? A radiological review following re-identification in Queensland. J. Med. Imaging Radiat. Oncol. 2020, 64, 229-235. [CrossRef] [PubMed]

7. Leung, C.C.; Yu, I.T.S.; Chen, W. Silicosis. Lancet 2012, 379, 2008-2018. [CrossRef]

8. Greenberg, M.I.; Waksman, J.; Curtis, J. Silicosis: A review. Dis. Mon. 2007, 53, 394-416. [CrossRef] [PubMed]

9. Lavoipierre, A. Silicosis Cases in Australia Rising, with Thousands Exposed to Unsafe Quantities of Silica in Past Decade 2019. Available online: https:/ / www.abc.net.au/news/2019-12-26/silicosis-cases-rapidly-climbing-more-toxic-asbestos-expert-says / 11826332 (accessed on 10 June 2020).

10. McMahon, A. Silicosis: Here's What You Need to Know about The Dust Lung Disease Killing Stonemasons 2018. Available online: https:/ / www.abc.net.au/news/2018-10-12/what-is-the-dust-lung-disease-silicosis/10365604 (accessed on 5 July 2020).

11. DNRME. Review of Respiratory Component of the Coal Mine Workers' Health Scheme for the Queensland Department of Natural Resources and Mines. Monash Centre for Occupational and Environmental Health, Monash University 2016. Available online: https:/ / www.dnrme.qld.gov.au/_data/assets/pdf_file/0009/383940/monash-qcwp-final-report-2016.pdf (accessed on 4 June 2020).

12. Palabiyik, S.S.; Girgin, G.; Tutkun, E.; Yilmaz, O.H.; Baydar, T. Immunomodulation and oxidative stress in denim sandblasting workers: Changes caused by silica exposure. Arh. Za Hig. Rada I Toksikologiju. 2013, 64, 431-437. [CrossRef]

13. Norboo, T.; Angchuk, P.T.; Yahya, M.; Kamat, S.R.; Pooley, F.D.; Corrin, B.; Kerr, I.H.; Bruce, N.; Ball, K.P. Silicosis in a Himalayan village population: Role of environmental dust. Thorax 1991, 46, 341-343. [CrossRef]

14. Gudmundsson, G. Respiratory health effects of volcanic ash with special reference to Iceland. A review. Clin. Respir. J. 2011, 5, 2-9. [CrossRef]

15. NIOSH. Health Effects of Occupational Exposure to Respirable Crystalline Silica; National Institute for Occupational Safety and Health (NIOSH), Department of Health and Human Services: Cincinnati, OH, USA, 2002.

16. Akgun, M.; Araz, O.; Akkurt, I.; Eroglu, A.; Alper, F.; Saglam, L.; Mirici, A.; Gorguner, M.; Nemery, B. An epidemic of silicosis among former denim sandblasters. Eur. Respir. J. 2008, 32, 1295-1303. [CrossRef] [PubMed]

17. Safe Work Australia. Crystalline Silica and Silicosis 2020. Available online: https://www.safeworkaustralia.gov.au/silica (accessed on 3 August 2020).

18. Raymond, L.W.; Wintermeyer, S. Medical Surveillance of Workers Exposed to Crystalline Silica. J. Occup. Environ. Med. 2006, 48, 95-101. [CrossRef] [PubMed]

19. RANZCR. Imaging of Occupational Lung Disease, Clinical Radiology, Position Statement. The Royal Australian and New Zealand College of Radiologists (RANZCR) 2019. Available online: https:/ /www.ranzcr.com/search/silicosis-position-statement (accessed on 3 August 2020).

20. Brilland, B.; Beauvillain, C.; Mazurkiewicz, G.; Rucay, P.; Roquelaure, Y.; Tabiasco, J.; Vinatier, E.; Riou, J.; Jeannin, P.; Renier, G.; et al. T Cell Dysregulation in Non-silicotic Silica Exposed Workers: A Step toward Immune Tolerance Breakdown. Front. Immunol. 2019, 10, 2743. [CrossRef] [PubMed]

21. Naha, N.; Muhamed, J.; Pagdhune, A.; Sarkar, B.; Sarkar, K. Club cell protein 16 as a biomarker for early detection of silicosis. Indian J. Med. Res. 2020, 151, 319-325. [PubMed]

22. RACP. Accelerated Silicosis. Royal Australasian College of Physicians (RACP) 2020. Available online: https://www.racp.edu. au/advocacy/division-faculty-and-chapter-priorities/faculty-of-occupational-environmental-medicine/accelerated-silicosis / faqs (accessed on 1 August 2020).

23. ILO. ILO International Classification of Radiographs of Pneumoconioses: International Labour Organization (ILO). 2011. Available online: https:/ / www.ilo.org/global/topics/safety-and-health-at-work/areasofwork/occupational-health/WCMS_108548/lang-en/index.htm (accessed on 6 April 2020).

24. NIOSH. Specific Medical Tests or Examinations Published in the Literature for OSHA-Regulated Substances 2014. Available online: https: / / www.cdc.gov/niosh/docs/2005-110/nmed0205.html (accessed on 24 August 2020).

25. NIOSH. Occupational Respiratory Disease Surveillance 2012. Available online: https://www.cdc.gov/niosh/topics/surveillance/ ords / workermedicalmonitoring.html (accessed on 24 August 2020).

26. Bandyopadhyay, A.; Majumdar, K.; Chakraborty, A.; Mitra, P.; Nag, S. CT-guided aspiration cytology of advanced silicosis and confirmation of the deposited zeolite nano particles through $X$ ray diffraction: A novel approach. Diagn. Cytopathol. 2016, 44, 246-249. [CrossRef] [PubMed] 
27. Lewis, L.; Fishwick, D. Health surveillance for occupational respiratory disease. Occup. Med. 2013, 63, 322-334. [CrossRef] [PubMed]

28. McGowan, J.; Straus, S.; Moher, D.; Langlois, E.V.; O’Brien, K.K.; Horsley, T.; Aldcroft, A.; Zarin, W.; Garitty, C.M.; Hempel, S.; et al. Reporting scoping reviews-PRISMA ScR extension. J. Clin. Epidemiol. 2020, 123, 177-179. [CrossRef] [PubMed]

29. Tricco, A.C.; Lillie, E.; Zarin, W.; O’Brien, K.K.; Colquhoun, H.; Levac, D.; Moher, D.; Peters, M.D.; Horsley, T.; Weeks, L.; et al. PRISMA Extension for Scoping Reviews (PRISMA-ScR): Checklist and Explanation. Ann. Intern. Med. 2018, 169, 467-473. [CrossRef]

30. Godin, K.; Stapleton, J.; Kirkpatrick, S.I.; Hanning, R.M.; Leatherdale, S.T. Applying systematic review search methods to the grey literature: A case study examining guidelines for school-based breakfast programs in Canada. Syst. Rev. 2015, 4, 138. [CrossRef] [PubMed]

31. Aggarwal, B.D. Lactate dehydrogenase as a biomarker for silica exposure-induced toxicity in agate workers. Occup. Environ. Med. 2014, 71, 578-582. [CrossRef]

32. Alexopoulos, E.C.; Bouros, D.; Dimadi, M.; Serbescu, A.; Bakoyannis, G.; Kokkinis, F.P. Comparative analysis of induced sputum and bronchoalveolar lavage fluid (BALF) profile in asbestos exposed workers. J. Occup. Med. Toxicol. 2011, 6, 23. [CrossRef] [PubMed]

33. Aslam, T.; Miele, A.; Chankeshwara, S.V.; Megia-Fernandez, A.; Michels, C.; Akram, A.R.; McDonald, N.; Hirani, N.; Haslett, C.; Bradley, M.; et al. Optical molecular imaging of lysyl oxidase activity-Detection of active fibrogenesis in human lung tissue. Chem. Sci. 2015, 6, 4946-4953. [CrossRef] [PubMed]

34. Chao, J.; Wang, X.; Zhang, Y.; Zhu, T.; Zhang, W.; Zhou, Z.; Yang, J.; Han, B.; Cheng, Y.; Tu, X.; et al. Role of MCPIP1 in the Endothelial-Mesenchymal Transition Induced by Silica. Cell. Physiol. Biochem. 2016, 40, 309-325. [CrossRef]

35. Chao, T.P.; Sperandio, E.F.; Ostolin, T.L.V.P.; Almeida, V.R.D.; Romiti, M.; Gagliardi, A.R.D.T.; Arantes, R.L.; Dourado, V.Z. Use of cardiopulmonary exercise testing to assess early ventilatory changes related to occupational particulate matter. Braz. J. Med. Biol. Res. 2018, 51. [CrossRef]

36. Chu, M.; Wu, S.; Wang, W.; Mao, L.; Yu, Y.; Jiang, L.; Yuan, W.; Zhang, M.; Sang, L.; Huang, Q.; et al. miRNA sequencing reveals miRNA-4508 from peripheral blood lymphocytes as potential diagnostic biomarker for silica-related pulmonary fibrosis: A multistage study. Respirology 2020, 25, 511-517. [CrossRef]

37. Chu, M.; Wu, S.; Wang, W.; Yu, Y.; Zhang, M.; Sang, L.; Tian, T.; Lu, Y.; Yuan, W.; Huang, Q.; et al. Functional variant of the carboxypeptidase $\mathrm{M}(\mathrm{CPM})$ gene may affect silica-related pneumoconiosis susceptibility by its expression: A multistage case-control study. Occup. Environ. Med. 2019, 76, 169-174. [CrossRef]

38. Codorean, E.; Raducanu, A.; Albulescu, L.; Tanase, C.; Meghea, A.; Albulescu, R. Multiplex cytokine profiling in whole blood from individuals occupationally exposed to particulate coal species. Rom. Biotechnol. Lett. 2011, 16, 6748-6759.

39. Corradi, M.; Gergelova, P.; Mutti, A. Use of exhaled breath condensate to investigate occupational lung diseases. Curr. Opin. Allergy Clin. Immunol. 2010, 10, 93-98. [CrossRef]

40. Cox, C.W.; Lynch, D.A. Medical imaging in occupational and environmental lung disease. Curr. Opin. Pulm. Med. 2015, 21, 163-170. [CrossRef] [PubMed]

41. Dinescu, V.C.; Puiu, I.; Dinescu, S.N.; Tudorascu, D.; Bica, E.C.; Vasile, R.C.; Bunescu, M.G.; Romanescu, F.M.; Cioatera, N.; Rotaru, L.T.; et al. Early predictive biochemical, electrocardiographic and echocardiographic markers for cardiac damage in patients with pulmonic silicosis. Rev. Chim. 2019, 70, 63-68. [CrossRef]

42. Ehrlich, R.I.; Myers, J.E.; Naude, J.M.T.W.; Thompson, M.L.; Churchyard, G.J. Lung function loss in relation to silica dust exposure in South African gold miners. Occup. Environ. Med. 2011, 68, 96-101. [CrossRef]

43. Greabu, M.; Didilescu, A.; Puiu, L.; Miricescu, D.; Totan, A. Salivary antioxidant biomarkers in non-ferrous metals mine workers-A pilot study. J. Oral Pathol. Med. 2012, 41, 490-493. [CrossRef]

44. Guo, L.; Ji, X.; Yang, S.; Hou, Z.; Luo, C.; Fan, J.; Ni, C.; Chen, F. Genome-wide analysis of aberrantly expressed circulating miRNAs in patients with coal workers' pneumoconiosis. Mol. Biol. Rep. 2013, 40, 3739-3747. [CrossRef]

45. Johnsen, H.L.; Bugge, M.D.; Føreland, S.; Kjuus, H.; Kongerud, J.; Søyseth, V. Dust exposure is associated with increased lung function loss among workers in the Norwegian silicon carbide industry. Occup. Environ. Med. 2013, 70, 803-809. [CrossRef]

46. Kahraman, H.; Koksal, N.; Cinkara, M.; Ozkan, F.; Sucakli, M.H.; Ekerbicer, H. Pneumoconiosis in dental technicians: HRCT and pulmonary function findings. Occup. Med. 2014, 64, 442-447. [CrossRef] [PubMed]

47. Kamaludin, N.H.; Jalaludin, J.; Tamrin, S.B.M.; Akim, A.M. Biomarker of occupational airways inflammation for exposure to inorganic dust. Future Food 2018, 6, 23-28.

48. Larici, A.R.; Mereu, M.; Franchi, P. Imaging in occupational and environmental lung disease. Curr. Opin. Pulm. Med. 2014, 20, 205-211. [CrossRef]

49. Lee, S.; Honda, M.; Yamamoto, S.; Kumagai-Takei, N.; Yoshitome, K.; Nishimura, Y.; Sada, N.; Kon, S.; Otsuki, T. Role of nephronectin in pathophysiology of silicosis. Int. J. Mol. Sci. 2019, 20, 2581. [CrossRef]

50. Lee, W.J.; Choi, B.S. Reliability and Validity of Soft Copy Images Based on Flat-panel Detector in Pneumoconiosis Classification. Comparison with the Analog Radiographs. Acad. Radiol. 2013, 20, 746-751. [CrossRef] [PubMed]

51. Lee, W.J.; Choi, B.S. Utility of digital radiography for the screening of pneumoconiosis as compared to analog radiography: Radiation dose, image quality, and pneumoconiosis classification. Health Phys. 2012, 103, 64-69. [CrossRef] [PubMed] 
52. Lee, W.J.; Choi, B.S.; Kim, S.J.; Park, C.K.; Park, J.S.; Tae, S.; Hering, K.G. Development of standard digital images for pneumoconiosis. J. Korean Med. Sci. 2011, 26, 1403-1408. [CrossRef] [PubMed]

53. Liu, S.J.; Wang, P.; Jiao, J.; Han, L.; Lu, Y.M. Differential gene expression associated with inflammation in peripheral blood cells of patients with pneumoconiosis. J. Occup. Health. 2016, 58, 373-380. [CrossRef] [PubMed]

54. Mao, L.; Laney, A.S.; Wang, M.K.; Sun, X.; Zhou, S.; Shi, J.; Shi, H. Comparison of digital direct readout radiography with conventional film-screen radiography for the recognition of pneumoconiosis in dust-exposed Chinese workers. J. Occup. Health 2011, 53, 320-326. [CrossRef]

55. Miao, R.; Ding, B.; Zhang, Y.; Xia, Q.; Li, Y.; Zhu, B. Proteomic profiling change during the early development of silicosis disease. J. Thorac. Dis. 2016, 8, 329-341. [CrossRef]

56. Nardi, J.; Nascimento, S.; Göethel, G.; Gauer, B.; Sauer, E.; Fao, N.; Cestonaro, L.; Peruzzi, C.; Souza, J.; Garcia, S.C. Inflammatory and oxidative stress parameters as potential early biomarkers for silicosis. Clin. Chim. Acta 2018, 484, 305-313. [CrossRef]

57. Okumura, E.; Kawashita, I.; Ishida, T. Development of CAD based on ANN analysis of power spectra for pneumoconiosis in chest radiographs: Effect of three new enhancement methods. Radiol. Phys. Technol. 2014, 7, 217-227. [CrossRef] [PubMed]

58. Ophir, N.; Shai, A.B.; Alkalay, Y.; Israeli, S.; Korenstein, R.; Kramer, M.R.; Fireman, E. Artificial stone dust-induced functional and inflammatory abnormalities in exposed workers monitored quantitatively by biometrics. ERS Monogr. 2016, 2, 00086-2015. [CrossRef]

59. Pelclová, D.; Fenclova, Z.; Vlckova, S.; Lebedova, J.; Syslová, K.; Pecha, O.; Belacek, J.; Navratil, T.; Kuzma, M.; Kacer, P. Leukotrienes B4, C4, D4 and E4 in the exhaled breath condensate (EBC), blood and urine in patients with pneumoconiosis. Ind. Health 2012, 50, 299-306.

60. Pelclova, D.; Fenclova, Z.; Syslova, K.; Vlčková, Š.; Lebedová, J.; Pecha, O.; Běláček, J.; Navrátil, T.; Kuzma, M.; Kačer, P. Oxidative stress markers in exhaled breath condensate in lung fibroses are not significantly affected by systemic diseases. Ind. Health 2011, 49, 746-754. [CrossRef]

61. Sato, T.; Saito, Y.; Inoue, S.; Shimosato, T.; Takagi, S.; Kaneko, T.; Ishigatsubo, Y. Serum heme oxygenase-1 as a marker of lung function decline in patients with chronic silicosis. J. Occup. Environ. Med. 2012, 54, 1461-1466. [CrossRef]

62. Sauni, R.; Oksa, P.; Lehtimäki, L.; Toivio, P.; Palmroos, P.; Nieminen, R.; Moilanen, E.; Uitti, J. Increased alveolar nitric oxide and systemic inflammation markers in silica-exposed workers. Occup. Environ. Med. 2012, 69, 256-260. [CrossRef] [PubMed]

63. Şener, M.U.; Şimşek, C.; Özkara, Ş.; Evran, H.; Bursali, İ.; Gökçek, A. Comparison of the international classification of highresolution computed tomography for occupational and environmental respiratory diseases with the international labor organization international classification of radiographs of pneumoconiosis. Ind. Health 2019, 57, 495-502. [CrossRef]

64. Sundararajan, R.; Xu, H.; Annangi, P.; Tao, X.; Sun, X.; Mao, L. A multiresolution support vector machine based algorithm for pneumoconiosis detection from chest radiographs 2010. In Proceedings of the 2010 IEEE International Symposium on Biomedical Imaging: From Nano to Macro, Rotterdam, The Netherlands, 14-17 April 2010.

65. Syslova, K.; Kačer, P.; Kuzma, M.; Pankracova, A.; Fenclova, Z.; Vlčková, Š.; Lebedova, J.; Pelclova, D. LC-ESI-MS/MS method for oxidative stress multimarker screening in the exhaled breath condensate of asbestosis/silicosis patients. J. Breath Res. 2010, 4, 017104. [CrossRef] [PubMed]

66. Trakultaweesuk, P.; Chaiear, N.; Boonsawat, W.; Khiewyoo, J.; Krisorn, P.; Silanun, K. Spirometry changes in normal or early ILO pneumoconiosis radiographs of sandstone-dust exposed workers: A preliminary result. J. Med. Assoc. Thail. 2017, 100, 1035-1044.

67. Uygur, F.; Ornek, T.; Tanriverdi, H.; Altuntas, M.; Altinsoy, B.; Erboy, F.; Tor, M.; Atalay, F. Platelet Indices in Patients with Coal Workers' Pneumoconiosis. Lung 2016, 194, 675-679. [CrossRef] [PubMed]

68. Weissman, D.N. Role of chest computed tomography in prevention of occupational respiratory disease: Review of recent literature. Semin. Respir. Crit. Care Med. 2015, 36, 433-448. [CrossRef]

69. Xing, J.; Huang, X.; Yang, L.; Liu, Y.; Zhang, H.; Chen, W. Comparison of high-resolution computerized tomography with film-screen radiography for the evaluation of opacity and the recognition of coal workers' pneumoconiosis. J. Occup. Health 2014, 56, 301-308. [CrossRef]

70. Xue, C.; Wu, N.; Li, X.; Qiu, M.; Du, X.; Ye, Q. Serum concentrations of Krebs von den Lungen-6, surfactant protein, D.; and matrix metalloproteinase-2 as diagnostic biomarkers in patients with asbestosis and silicosis: A case-control study. BMC Pulm. Med. 2017, 17, 144. [CrossRef]

71. Yang, H.-Y.; Shie, R.-H.; Chang, C.-J.; Chen, P.-C. Development of breath test for pneumoconiosis: A case-control study. Respir. Res. 2017, 18, 1-8. [CrossRef]

72. Young, C.; Barker, S.; Ehrlich, R.; Kistnasamy, B.; Yassi, A. Computer-aided detection for tuberculosis and silicosis in chest radiographs of gold miners of South Africa. Int. J. Tuberc. Lung Dis. 2020, 24, 444-451. [CrossRef] [PubMed]

73. Yu, P.; Xu, H.; Zhu, Y.; Yang, C.; Sun, X.; Zhao, J. An Automatic Computer-Aided Detection Scheme for Pneumoconiosis on Digital Chest Radiographs. J. Digit. Imaging 2011, 24, 382-393. [CrossRef] [PubMed]

74. Zhang, G.H.; Li, L.; Hao, C.; Ren, J.C.; Zhang, H.; Jiao, J.; Gao, L.; Ding, S.; Yao, S.; Yao, W.; et al. Screening and Preliminary Verification of a Phage Display Single-Chain Antibody Library Against Coal Workers' Pneumoconiosis. J. Occup. Environ. Med. 2016, 58, 1264-1269. [CrossRef]

75. Zhao, W.; Xu, R.; Hirano, Y.; Tachibana, R.; Kido, S.; Suganuma, N. Classification of pneumoconiosis on HRCT images for computer-aided diagnosis. IEICE Trans. Inf. Syst. 2013, 96, 836-844. [CrossRef] 
76. Zhu, L.; Zheng, R.; Jin, H.; Zhang, Q.; Zhang, W. Automatic detection and recognition of silicosis in chest radiograph. Bio.-Med. Mater. Eng. 2014, 24, 3389-3395. [CrossRef]

77. TSANZ. Standards for Spirometry Training Courses Companion Document to Standards for the Delivery of Spirometry for Coal Mine Workers. Thoracic Society of Australia and New Zealand (TSANZ) 2017. Available online: https:/ /www.dnrme.qld.gov. au/_data/assets/pdf_file/0004/1274422/tsanz-spirometry-training-standards.pdf (accessed on 22 June 2020).

78. Tamura, T.; Suganuma, N.; Hering, K.G.; Vehmas, T.; Itoh, H.; Akira, M.; Takashima, Y.; Hirano, H.; Kusaka, Y. Relationships (I) of International Classification of High-resolution Computed Tomography for Occupational and Environmental Respiratory Diseases with the ILO International Classification of Radiographs of Pneumoconioses for parenchymal abnormalities. Ind. Health 2015, 53, 260-270. [CrossRef]

79. Newbigin, K.; Parsons, R.; Deller, D.; Edwards, R.; McBean, R. Stonemasons with silicosis: Preliminary findings and a warning message from Australia. Respirology 2019, 24, 1220-1221. [CrossRef] [PubMed]

80. Cox, C.W.; Rose, C.S.; Lynch, D.A. State of the art: Imaging of occupational lung disease. Radiology 2014, 270, 681-696. [CrossRef] [PubMed]

81. Bacchus, L.; Shah, R.D.; Chung, J.H.; Crabtree, T.P.; Heitkamp, D.E.; Iannettoni, M.D.; Johnson, G.B.; Jokerst, C.; McComb, B.L.; Saleh, A.G.; et al. ACR Appropriateness Criteria Review ACR Appropriateness Criteria ${ }^{\circledR}$ Occupational Lung Diseases. J. Thorac. Imaging 2016, 31, W1-W3. [CrossRef]

82. Sies, H. Oxidative stress: Oxidants and antioxidants. Exp. Physiol. 1997, 82, 291-295. [CrossRef]

83. Schulte, P.A. The use of biomarkers in surveillance, medical screening, and intervention. Mutat. Res./Fundam. Mol. Mech. Mutagen. 2005, 592, 155-163. [CrossRef]

84. Thakkar, L.R.; Pingle, S.K.; Tumane, R.G.; Soni, P.N. Expression of Low Molecular Mass Proteins in Stone Quarry Workers. Natl. J. Lab. Med. 2013, 2, 8-11.

85. Pandey, J.K.; Agarwal, D. Biomarkers: A potential prognostic tool for silicosis. Indian J. Occup. Environ. Med. 2012, 16, 101-107. [CrossRef]

86. Horváth, I.; Barnes, P.J.; Loukides, S.; Sterk, P.J.; Högman, M.; Olin, A.C.; Amann, A.; Antus, B.; Baraldi, E.; Bikov, A.; et al. A European Respiratory Society technical standard: Exhaled biomarkers in lung disease. Eur. Respir. J. 2017, 49, 1600965. [CrossRef] [PubMed]

87. Leese, E.; Staff, J.F.; Carolan, V.A.; Morton, J. Exhaled Breath Condensate: A Novel Matrix for Biological Monitoring to Assess Occupational Exposure to Respirable Crystalline Silica. Ann. Work. Expo. Health 2017, 61, 902-906. [CrossRef] [PubMed] 